\title{
Targeting neural synchrony deficits is sufficient to improve cognition in a schizophrenia-related neurodevelopmental model
}

\author{
Heekyung Lee ${ }^{1 t \neq}$, Dino Dvorak ${ }^{2 \ddagger}$ and André A. Fenton ${ }^{3,4 *}$ \\ 1 Graduate Program in Neural and Behavioral Science, Downstate Medical Center, State University of NewYork, Brooklyn, NY, USA \\ ${ }^{2}$ Graduate Program in Biomedical Engineering, Downstate Medical Center, State University of New York and New York University Polytechnic School of Engineering, \\ Brooklyn, NY, USA \\ ${ }^{3}$ The Robert F. Furchgott Center for Neural and Behavioral Science, Downstate Medical Center, State University of New York, Brooklyn, NY, USA \\ ${ }^{4}$ Center for Neural Science, New York University, New York, NY, USA
}

\section{Edited by:}

André Schmidt, University of Basel, Switzerland

\section{Reviewed by:}

Vaibhav A. Diwadkar, Wayne State University School of Medicine, USA John Gigg, University of Manchester, UK

\section{*Correspondence:}

André A. Fenton, Center for Neural

Science, New York University, 4

Washington Place, New York, NY

10003, USA

e-mail: afenton@nyu.edu

\section{${ }^{\dagger}$ Present address:}

Heekyung Lee, Krieger Mind/Brain Institute, Johns Hopkins University, Baltimore, MD, USA

${ }^{\ddagger}$ Heekyung Lee and Dino Dvorak have contributed equally to this work.
Cognitive symptoms are core features of mental disorders but procognitive treatments are limited. We have proposed a "discoordination" hypothesis that cognitive impairment results from aberrant coordination of neural activity. We reported that neonatal ventral hippocampus lesion (NVHL) rats, an established neurodevelopmental model of schizophrenia, have abnormal neural synchrony and cognitive deficits in the active place avoidance task. During stillness, we observed that cortical local field potentials sometimes resembled epileptiform spike-wave discharges with higher prevalence in NVHL rats, indicating abnormal neural synchrony due perhaps to imbalanced excitation-inhibition coupling. Here, within the context of the hypothesis, we investigated whether attenuating abnormal neural synchrony will improve cognition in NVHL rats. We report that: (1) inter-hippocampal synchrony in the theta and beta bands is correlated with active place avoidance performance; (2) the anticonvulsant ethosuximide attenuated the abnormal spike-wave activity, improved cognitive control, and reduced hyperlocomotion; (3) ethosuximide not only normalized the task-associated theta and beta synchrony between the two hippocampi but also increased synchrony between the medial prefrontal cortex and hippocampus above control levels; (4) the antipsychotic olanzapine was less effective at improving cognitive control and normalizing place avoidance-related inter-hippocampal neural synchrony, although it reduced hyperactivity; and (5) olanzapine caused an abnormal pattern of frequency-independent increases in neural synchrony, in both NVHL and control rats. These data suggest that normalizing aberrant neural synchrony can be beneficial and that drugs targeting the pathophysiology of abnormally coordinated neural activities may be a promising theoretical framework and strategy for developing treatments that improve cognition in neurodevelopmental disorders such as schizophrenia.

Keywords: neural synchrony, oscillations, schizophrenia, neurodevelopmental models, hippocampus, local field potentials, cognitive control, spike-wave discharge

\section{INTRODUCTION}

Cognitive impairment is a core feature of schizophrenia but treatments to improve cognition are limited, in part because the physiological mechanisms of cognitive dysfunction are unclear. Recent interest has been directed toward understanding the abnormal synchrony of neural oscillations as a pathophysiological mechanism that underlies the cognitive impairments in schizophrenia (1-4). Dysfunctional neural synchrony in a wide range of frequencies, including the delta (5), theta (6), beta (7), and gamma ranges $(8,9)$, have been characterized in patients with schizophrenia. In animal models, disruption of long-range hippocampalprefrontal synchrony has been reported while performing a working memory task in a genetic mouse model of schizophrenia (10) and during an open-field task in the maternal immune activation (MIA) neurodevelopmental model of schizophrenia (11). Abnormal hippocampal-prefrontal synchrony was also observed in the neonatal ventral hippocampus lesion (NVHL) neurodevelopmental model but this synchrony abnormality was not itself associated with cognitive impairment (12). In fact, aberrant neural synchrony may be a common pathophysiology responsible for impaired cognition in a variety of disorders $(2,13)$. Indeed, treatments that normalize neural network dysfunction without addressing the underlying cellular pathology have been sufficient to improve cognitive function in animal models of neurodegenerative diseases $(14,15)$. Such studies demonstrate it may be a feasible strategy to improve cognitive functional outcomes by normalizing aberrant neural network synchrony in cases where the network dysfunction is part of the causal chain from the disease etiology to the manifest cognitive symptoms.

We previously reported that NVHL rats, an established neurodevelopmental animal model of schizophrenia (16), have a cognitive control impairment that was associated with abnormal 
synchrony between the two hippocampi (12). We also detected hippocampal-prefrontal synchrony abnormalities in these rats during home-cage resting behaviors, but these abnormalities were absent during place avoidance training, and so not related to performance on the cognitive test. Clinically, the presence of hippocampal dysfunction is apparent in schizophrenia. Postmortem and neuroimaging studies indicate that the hippocampus is one of the brain regions most consistently altered in schizophrenia (17-22). Furthermore, functional magnetic resonance imaging studies show dysfunctional hippocampal activities are associated with poor cognitive performance in schizophrenia patients (23-25). Perhaps similar to the findings in NVHL rats, reduced inter-hippocampal functional coupling has also been observed between the left- and right-hippocampus areas in healthy carriers of the CACNA1C risk variant in bipolar disorder (26), but to our knowledge these measurements have not been carried out in schizophrenia subjects. Because neuroimaging studies do not have sufficient temporal resolution to detect functional synchrony at the 100 -ms or faster timescales of the electrophysiological synchrony that underlies information processing between neural networks, it is unclear whether or not the inter-hippocampal abnormalities in NVHL rats mimics abnormalities in schizophrenia. Regardless, it is our opinion that animal models used to study schizophrenia do not sensibly mimic disease features, instead they are better thought of as being models for testing hypotheses that are relevant to understanding schizophrenia. Thus, we hypothesized that the abnormal hippocampal synchrony plays a role in the pathophysiology of cognitive control impairment in a schizophrenia-related neurodevelopmental animal model. As outlined below, in the present study, we tested the idea that attenuating this pathophysiology can improve cognition.

We, like others, have proposed the discoordination hypothesis, which asserts that cognitive impairment arises because of aberrant coordination of what may otherwise be normal neural activities $(12,27)$. The discoordination hypothesis is rooted in the idea that disrupted physiological coordination and synchronization of unitary neural processes impair the ability to selectively and dynamically activate and suppress information for organizing knowledge and perception into useable representations (1, 2, 27, 28). We use the term (dis)coordination to refer to the collective temporal organization of unitary neural activity such as action and synaptic and local field potentials (LFPs) at individual sites. Thus, (dis)coordination can be measured at different temporal and spatial scales. In the present work, we examined inter-hippocampal synchrony of LFP oscillations. These oscillations are an example of a long-range spatial scale neural interaction that involves an approximate order of $10^{5}$ neurons. These oscillations result from the multiple timescales of excitation-inhibition neural activity cycles that govern spiking within these networks (29). The discoordination hypothesis predicts that in the NVHL model, restoring the abnormal neural synchrony to normal will attenuate the cognitive control deficit. Accordingly, we took advantage of the hippocampal synchrony abnormality in NVHL rats, specifically the inter-hippocampal synchrony that was associated with cognitive performance, to evaluate the prediction. We examined whether normalizing the abnormal synchrony can attenuate the cognitive control deficit in the NVHL rats. We observed that hippocampal and neocortical LFPs in the NVHL rats sometimes resembled spike-wave discharges (SWDs), a sign of network hyperexcitability. We hypothesized that these aberrant neural activities arise from an excitation-inhibition imbalance that may underlie abnormal neural synchrony in the NVHL rats. Since SWD in epilepsy patients is treated with ethosuximide (2-ethyl-2-methylsuccinimide), an anticonvulsant that acts by blocking T-type $\mathrm{Ca}^{2+}$ channels, we studied whether ethosuximide would attenuate the abnormal SWD-like synchrony as well as impaired cognitive behavior and the associated neural synchrony that we have previously described in the NVHL model (12). We stress that our goal is to test the prediction of the discoordination hypothesis, not to suggest the use of ethosuximide as an antipsychotic. For comparison, we also examined the effects of the atypical antipsychotic olanzapine on neural synchrony and cognitive abnormalities in NVHL rats. Given that antipsychotics are not known to improve cognitive symptoms, the hypothesis predicts that olanzapine will not correct either the neural synchrony or cognitive abnormalities in NVHL rats.

We report that in NVHL rats, ethosuximide treatment attenuated the abnormal spike-wave activity, and most importantly, ethosuximide normalized the cognition-associated synchrony of theta and beta oscillations between the two hippocampi, and improved cognitive control in the place avoidance task. Olanzapine was less effective at reducing the abnormal spike-wave activity and normalizing the synchrony of oscillations between the two hippocampi and it did not improve cognitive control in NVHL rats, although it attenuated hyperlocomotion during the cognitive testing. These data are consistent with the discoordination hypothesis, and support the idea that drugs targeting the abnormal neural synchrony may be sufficient to rescue the cognitive deficits in schizophrenia-related neurodevelopmental animal models.

\section{MATERIALS AND METHODS}

All procedures were in compliance with National Institutes of Health guidelines and were approved by Downstate Medical Center's Institutional Animal Care and Use Committee.

\section{NEONATAL VENTRAL HIPPOCAMPAL LESION}

The procedure followed the manual provided by Lipska and Weinberger. We used the exact protocol that is published in our prior work (12). Briefly, timed-pregnant (13 or 14 days in gestation) female Long-Evans rats were obtained from Charles River Laboratories (Wilmington, MA, USA). Pups were born at the Downstate animal facility. On postnatal day 7 (P7), male pups were anesthetized by hypothermia. Bilateral puncture holes (relative to bregma AP: $-3.0 \mathrm{~mm}$, ML: $\pm 3.5 \mathrm{~mm}$ ) were made in the skull with a $30-\mathrm{Ga}$ injection needle. A bilateral infusion $(0.3 \mu \mathrm{l} / \mathrm{side})$ of ibotenic acid solution $(10 \mu \mathrm{g} / \mu \mathrm{l})$ was delivered to each ventral hippocampus (relative to the skull surface DV: $-5.0 \mathrm{~mm}$ ). Control animals were treated identically except saline was injected instead of ibotenic acid. A subset of the NVHL and control rats were given drug treatments as described below. The behavioral and neural synchrony measurements from these rats were compared to untreated NVHL and control rats, the data from which were reported previously (12), although all data collection was concurrent amongst the different treatment groups. 


\section{DRUGS}

Ibotenic acid, purchased from Sigma (St. Louis, MO, USA), was dissolved in artificial cerebrospinal fluid (ACSF) to a final concentration of $10 \mu \mathrm{g} / \mu \mathrm{l}, \mathrm{pH}$ 7.6. Ethosuximide, purchased from Sigma (St. Louis, MO, USA), was dissolved in sterile $0.9 \% \mathrm{NaCl}$ and a concentration of $100 \mathrm{mg} / \mathrm{kg}$ was injected i.p. Olanzapine, purchased from Toronto Research Chemicals Inc. (Toronto, ON, Canada), was dissolved in dimethyl sulfoxide (DMSO) and $1 \mathrm{mg} / \mathrm{kg}$ was injected i.p. Both ethosuximide and olanzapine were administered just prior to the start of behavioral training (see below) and based on published work, they were expected to have been bioactive for several hours, beyond the duration of behavioral training.

\section{TWO-FRAME AVOIDANCE TASK}

Five adult rats (P65) per group were trained on the two-frame task. The rat was placed on a circular $82 \mathrm{~cm}$ diameter, stainless steel rotating arena (Bio-Signal Group Corp., Brooklyn, NY, USA). The rat's position was tracked using an overhead camera and software (Tracker, Bio-Signal Group Corp., Brooklyn, NY, USA). All animals were habituated to the rotating arena the day before the start of active place avoidance training during two 10-min sessions without any shocks. On the following training trials, whenever the rat on the rotating arena entered a $60^{\circ}$ shock zone that was defined in room coordinates, a mild constant current $(60 \mathrm{~Hz},<0.4 \mathrm{~mA}$, $500 \mathrm{~ms}$ ) foot shock was delivered. Foot shock was repeated every $1.5 \mathrm{~s}$ until the rat left the shock zone. Each place avoidance trial was $10 \mathrm{~min}$ and the interval between the trials was at least $10 \mathrm{~min}$. A total of eight training trials were given on the training day. The drugs were injected i.p. just prior to the first avoidance training trial. Avoidance was measured by counting the number of times the rat entered the shock zone.

\section{LOCAL FIELD POTENTIAL AND ELECTROENCEPHALOGRAM RECORDINGS}

All rats were housed individually, exposed to a 12-h light/dark circadian cycle, and had ad libitum access to water and food. When rats were P55-57, electrodes were surgically implanted bilaterally. Rats were given post-operative Ketofen $(5 \mathrm{mg} / \mathrm{kg})$ for 2 days after surgery and allowed to recover from the surgical procedure for 7 days. LFPs in the dorsal hippocampus (AP: $-4 \mathrm{~mm}$; ML: $\pm 2.5 \mathrm{~mm}$; DV: $3 \mathrm{~mm}$ ) and in the medial prefrontal cortex (mPFC; AP: $+3 \mathrm{~mm}$; ML: $\pm 1 \mathrm{~mm}$; DV: $4 \mathrm{~mm}$ ) were recorded by implanting 75- $\mu \mathrm{m}$ Nichrome wires that were attached to a Millmax connector. The epidural cortical electroencephalogram (EEG) was recorded with a screw electrode in the frontal and parietal bones of each hemisphere. All electrodes were referred to an electrode implanted in the cerebellar white matter (AP: $-10 \mathrm{~mm}$; ML: $+2 \mathrm{~mm}$; DV: $3 \mathrm{~mm}$ ), which seemed to be normal in NVHL rats. Recordings were made with a wireless digital telemetry system (Bio-Signal Group, Brooklyn, NY, USA). Recordings were made while the rats were performing the two-frame avoidance task and in the home-cage (for 2-3 h) during the light cycle.

The signals at the electrode connector were amplified (300 times), low-pass filtered $(6 \mathrm{kHz})$ and digitized (24-bits, $12 \mathrm{kHz}$ using delta-sigma analog-digital convertors). The digital signals were transmitted wirelessly to a recording system (dacqUSB, Axona Ltd., St. Albans, UK) for band-pass filtering $(1-500 \mathrm{~Hz})$, digital amplification and down-sampling (16-bits, $2000 \mathrm{~Hz}$ ) using digital signal processors. The digital electrophysiological data were stored on computer hard drives for off-line analysis. The channels were marked if they had saturated signals and were excluded from further analysis if the proportion was substantial. At the end of training and recording, the rats were trans-cardially perfused and the brains removed for histological verification of the electrode locations.

\section{POWER SPECTRA}

Power spectra measure the amplitude of frequency-specific oscillations that arise when spatially patterned synaptic potentials are coincident in time. These oscillations are locally generated phenomena. As such, an oscillation itself may not be a strong indication of neural synchrony as it relates to the concept of neural coordination $(1,27)$. Instead, power spectra are an estimate of the amount and magnitude of patterns of oscillation-generating synaptic potentials that are the fundamental components of neural coordinating processes. The patterns of synaptic potentials must themselves be temporally and spatially coordinated within and between neural networks to mediate the neural computations and information processing upon which cognition is based (29).

To compute power spectra, LFP signals were first normalized by dividing the signal by its RMS value. This step minimized electrode-specific signal properties caused by variability in electrode location or impedance. The signal was processed using $5 \mathrm{~s}$ long non-overlapping windows. For each window power spectral density (PSD) was computed using the "pwelch" Matlab function. The spectral peak at $60 \mathrm{~Hz}$ was removed and the PSD was interpolated between 58 and $62 \mathrm{~Hz}$. The resulting PSD was normalized by dividing it by its sum so the total power was equal to 1 . Based on the spectral profile, we then defined bands of interest for frequency-specific phase-locking analysis. We excluded the delta band $(0-3 \mathrm{~Hz})$ because of its attenuation by the hardware DC-removal filter (Figure 1). We defined the theta band as $5-15 \mathrm{~Hz}$, beta as $20-30 \mathrm{~Hz}$ in order to exclude the strong second theta harmonic (Figure 1), slow gamma $30-55 \mathrm{~Hz}$ and fast gamma as $65-100 \mathrm{~Hz}$ (excluding the $60-\mathrm{Hz}$ region). Figure 1 shows that the left and right hippocampal spectra are similar, as expected, and that the spectra are attenuated in NVHL rats. Both ethosuximide and olanzapine only caused a marginal increase in the broadband spectral power. This confirmation of stable spectral power provides the starting point for the present study, which focuses on measuring how these component oscillations are coordinated between brain regions.

\section{PHASE-LOCKING VALUE}

The coordination of neural signals at a pair of electrodes was estimated by computing the phase-locking value (PLV). Eight LFP channel pairs were selected for PLV analysis. They were the leftand right-hippocampi; left- and right-mPFC; left-hippocampus and left-mPFC; right-hippocampus and right-mPFC, as well as the inter-hemispheric and associational epidural electrode pairs. The PLV at a pair of electrodes was computed using custom software written in Matlab, based on a published algorithm (31). The phase of a signal at time $t$ and sample $n \phi(t, n)$ was obtained by filtering the signal with a narrow-band finite input response (FIR) 

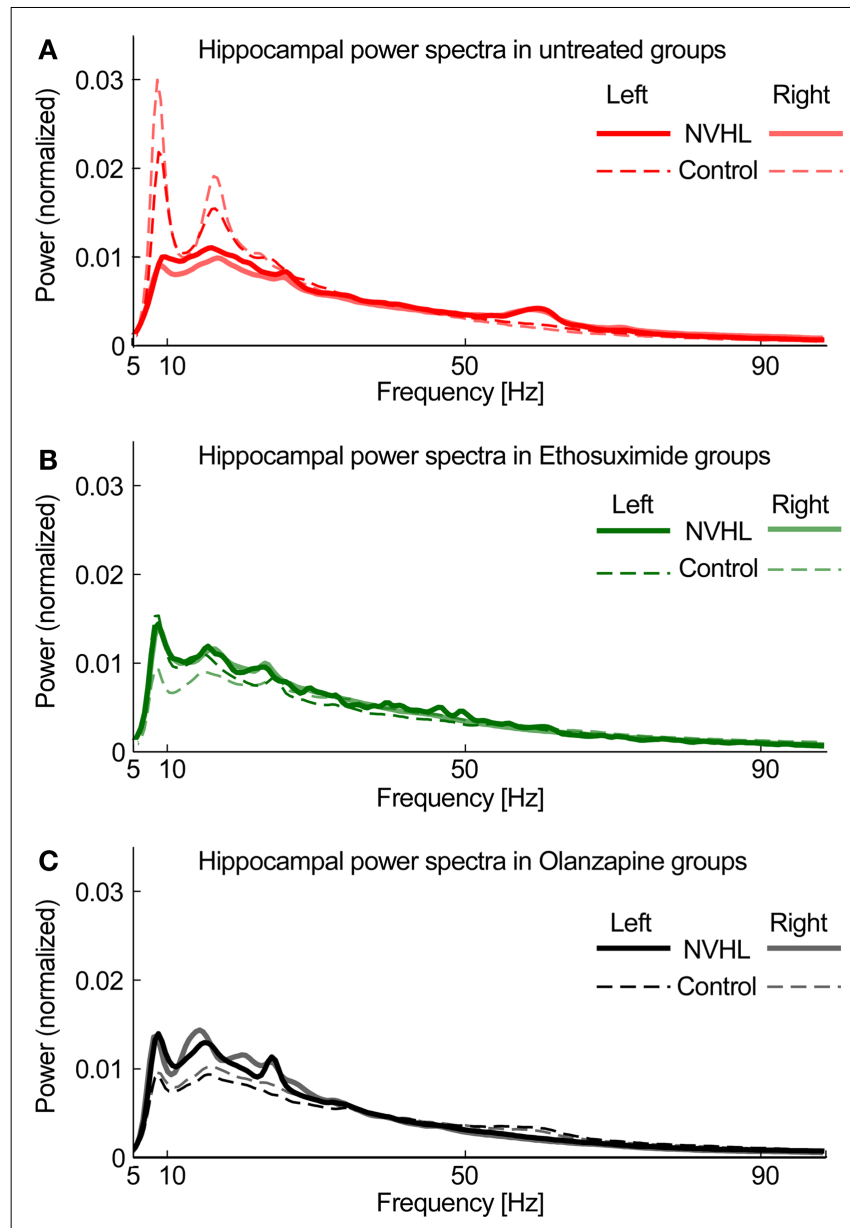

FIGURE 1 | Hippocampal power spectra. (A) Power spectra density plot for untreated groups averaged across all subjects for NVHL (thick line) and control (thin line) computed independently for left (opaque line) and right (semi-transparent line) hippocampi. (B) Power spectral density plot for Ethosuximide-treated animals. (C) Power spectra density plot for Olanzapine-treated animals. The similarities between the corresponding left and right spectra is a common observation, is independent of phase synchrony, and is only indirectly related to neural coordination in that neural oscillations are the fundamental, unitary components of neural coordination processes such as phase synchrony and cross-frequency coupling (30).

filter using a zero phase-shift filtering algorithm followed by the Hilbert Transform. The filters were designed using the Matlab filter design toolbox. Filters in the range $0-100 \mathrm{~Hz}$ had fixed bandwidth of $5 \mathrm{~Hz}$. For bands of interest extending over several $5 \mathrm{~Hz}$ bands the PLV across all such bands were averaged. Given a pair of LFP signals of $N$ samples, the PLV was defined as follows:

$$
\operatorname{PLV}=\frac{1}{N} \mid \sum_{n=0}^{N} \exp [i \theta(t, n)],
$$

where $\theta(t, n)$ is the phase difference $\phi_{1}(t, n)-\phi_{2}(t, n)$ between the two signals at time $t$ sample $n$; $N$ is the total number of samples; and $i$ is the imaginary unit. Prior work showed that the intracranial electrode-pair specific PLVs at the four (theta, beta, slow gamma, fast gamma) frequency bands were different between NVHL and control rats (12). We made these comparisons between the NVHL and control treatment groups to determine if the drug treatment normalized neural synchrony between the particular electrode pairs. In addition, the PLVs for the full set of electrode pairs were studied to look for neural synchrony patterns that distinguished the NVHL and control and the drug-treatment groups. The set of electrode-pair and frequency-specific PLVs for each recording session was treated as a vector with dimension 32 (8 electrode pairs $\times 4$ bands). Principal component analysis (PCA) was performed to reduce the dimensionality and identify the most useful measures of synchrony. Each session vector of PLVs (eight sessions per rat) was plot in the coordinate system of the first three principal components to visualize the group patterns. The first six principal components explained over $90 \%$ of the variance and so for quantification we only considered the first six principal components. We quantified the distance between the vectors in each session group by computing the 6-D Euclidean distance between all pairs of data points within a treatment group, and also between all pairs of data points against a reference treatment group. The untreated control and untrained NVHL groups as well as the ethosuximidetreated NVHL and the olanzapine-NVHL groups each served as reference groups for the comparisons. These pair-wise group distances were compared by two-sample Student's $t$-tests that were adjusted for multiple comparisons using the Bonferroni method.

\section{AUTOMATIC DETECTION OF SPIKE-WAVE ACTIVITY}

We used an algorithm to identify activity that resembled epileptiform SWDs, but are observed in normal Long-Evans rats without seizures (32). Each channel was first examined for signal saturation and only data segments with brief $(<50 \mathrm{~ms})$ saturations were considered for further analysis. Brief saturations in the LFP were allowed so spike-wave complexes with a large spike component were not removed from the analysis. We manually marked epochs of spike-wave events using the EEGLAB toolbox for Matlab. These epochs were used to calculate and optimize the detection parameters for the automatic classifier of spike-wave events. The classifier is a modified version of a published algorithm (33).

The algorithm worked in several steps (Figure 2):

(1) Local field potential signals (Figure 2A) were transformed (Figure 2B) using the continuous wavelet transform (CWT) from the Wavelet Toolbox in Matlab (Morlet wavelets, scales 5-50 with step of 5 corresponding to pseudo-frequencies $16-160 \mathrm{~Hz}$ ).

(2) The sliding variance (50 ms long window) was computed for each scale and all variances were summed across all scales resulting in a single variance profile over time.

(3) Candidate spike-wave events were marked as threshold crossings (red line in Figure 2C) of the summed variance series.

(4) Gaps between individual marked segments shorter than $0.75 \mathrm{~s}$ were filled to create longer segments.

(5) After filling the gaps, all marked segments that were shorter than $1 \mathrm{~s}$ were removed from further analysis.

(6) Each marked segment was tested for periodic spiking activity by detecting local maxima (red dots in Figure 2C) in the summed variance signal with $50 \mathrm{~ms}$ smoothing applied. The 


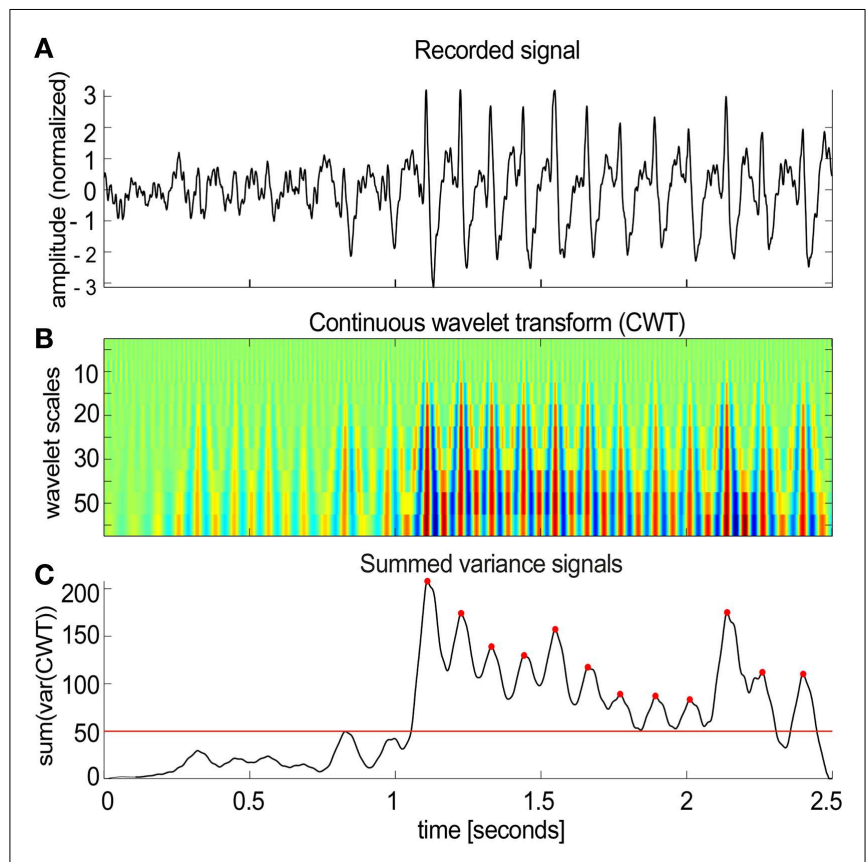

FIGURE 2 | Spike-wave activities in NVHL and control rats

(A) A segment of the cortical EEG with spike-wave activity. An automatic classifier was used to detect these events. (B) The first step in the classifier was to transform the recorded signal using the continuous wavelet transform (CWT). (C) Then a sliding variance was computed for each wavelet scale. All the variances were summed across all scales resulting in a single variance profile over time $(50 \mathrm{~ms}$ smoothing was applied to the resulting signal). SWD candidates were detected as threshold crossings [red line in (C)]. Each marked segment was tested for periodic spiking activity by detecting local maxima [red dots in (C)]. Only the detected segments that contained at least $50 \%$ of inter-spike intervals in the range of $2-10 \mathrm{~Hz}$ were included in further analysis.

inter-spike intervals for each pair of sequential maxima were computed. Only those marked segments that contained at least $50 \%$ of inter-spike intervals in the range of $2-10 \mathrm{~Hz}$ (the typical range of SWDs) were considered as spike-wave segments.

(7) The process was repeated several times with different parameters and the classified event segments were compared with manually marked events to tune the classifier.

\section{ANALYSIS OF SPIKE-WAVE EVENT FEATURES}

Only manually marked segments were included in this analysis to avoid distortion by borderline spike-wave-like events that were detected by the automatic algorithm but did not necessarily share all the features such as clear spike and wave components. The subjectively selected events were identified on the basis of the classic spike-wave morphology, by an observer blind to the origin of the EEG. Thus the manual selection of spike-wave events resulted in a dataset of events with a bias to less specificity and more selectivity.

The manually marked events were first low-pass filtered with a cut-off frequency of $100 \mathrm{~Hz}$. The filter removed the high frequency components that could cause errors in feature extraction. Local maxima were then detected in the signal and for each maximum, its preceding and following local minima were found. The spike components of the events were then defined as local maxima, surrounded by local minima with a maxima-minima amplitude of at least $400 \mu \mathrm{V}$ and with a spike width (defined below) of at least $50 \mathrm{~ms}$. Spike sequences (for computing the inter-spike time) were defined as continuous spikes with a frequency between 5 and $13 \mathrm{~Hz}$. The feature analysis was repeated several times to optimize the parameters for the component detection.

The following features were computed for both NVHL and control rats (Figure 3):

(1) Spike duration: the duration of the spike measured at the level of either the preceding or the following local minimum with the higher voltage.

(2) Spike-to-valley voltage: the voltage between the spike peak and the following local minimum (valley).

(3) Inter-spike time: the time between two successive spikes.

(4) Wave duration: the duration of the wave component measured from the local minimum (valley) following the spike component and the local minimum preceding the next spike component.

(5) Wave energy: the energy of the wave component computed as the sum of squares of amplitudes.

(6) Spike-wave event prevalence: only the automatically classified segments were included in this analysis. Prevalence was estimated as the total time spike-wave events were detected and divided by the total amount of time that was analyzed.

\section{STATISTICAL ANALYSIS}

Group differences were compared by ANOVA or Student's $t$-test. The PLV between groups and across frequency bands were compared by two-way ANOVA. Repeated-measures were not used because the data were independently and differentially filtered for computing the synchrony estimates at each frequency band. Significance was set at $p<0.05$, and Tukey's HSD post hoc tests were used as appropriate. The two-sample Kolmogorov-Smirnov test was used to compare the probability distributions of spike-wave event features in the NVHL and control groups. The distributions were generated by selecting a constant subsample of spike-wave events from each animal such that each subject contributed equally to the distribution. The resulting distribution was then divided by its sum to generate probability distributions that are normalized against different numbers of subjects in the NVHL and control groups.

\section{RESULTS}

\section{ABNORMAL SPIKE-WAVE ACTIVITIES IN THE EEG OF NVHL RATS}

Before focusing on the main aim of this study, to investigate cognition-related inter-hippocampal phase synchrony during active place avoidance cognitive behavior, we began by monitoring the field potential recordings from NVHL and control rats as they behaved in their home-cage. In the home-cage, both groups of rats were still or moving $<0.5 \mathrm{~cm} / \mathrm{s}$ about $80 \%$ of the time based on estimates from concurrent video tracking. The most conspicuous feature was the occasional presence of a large amplitude series of spike-wave events. We were surprised to find 

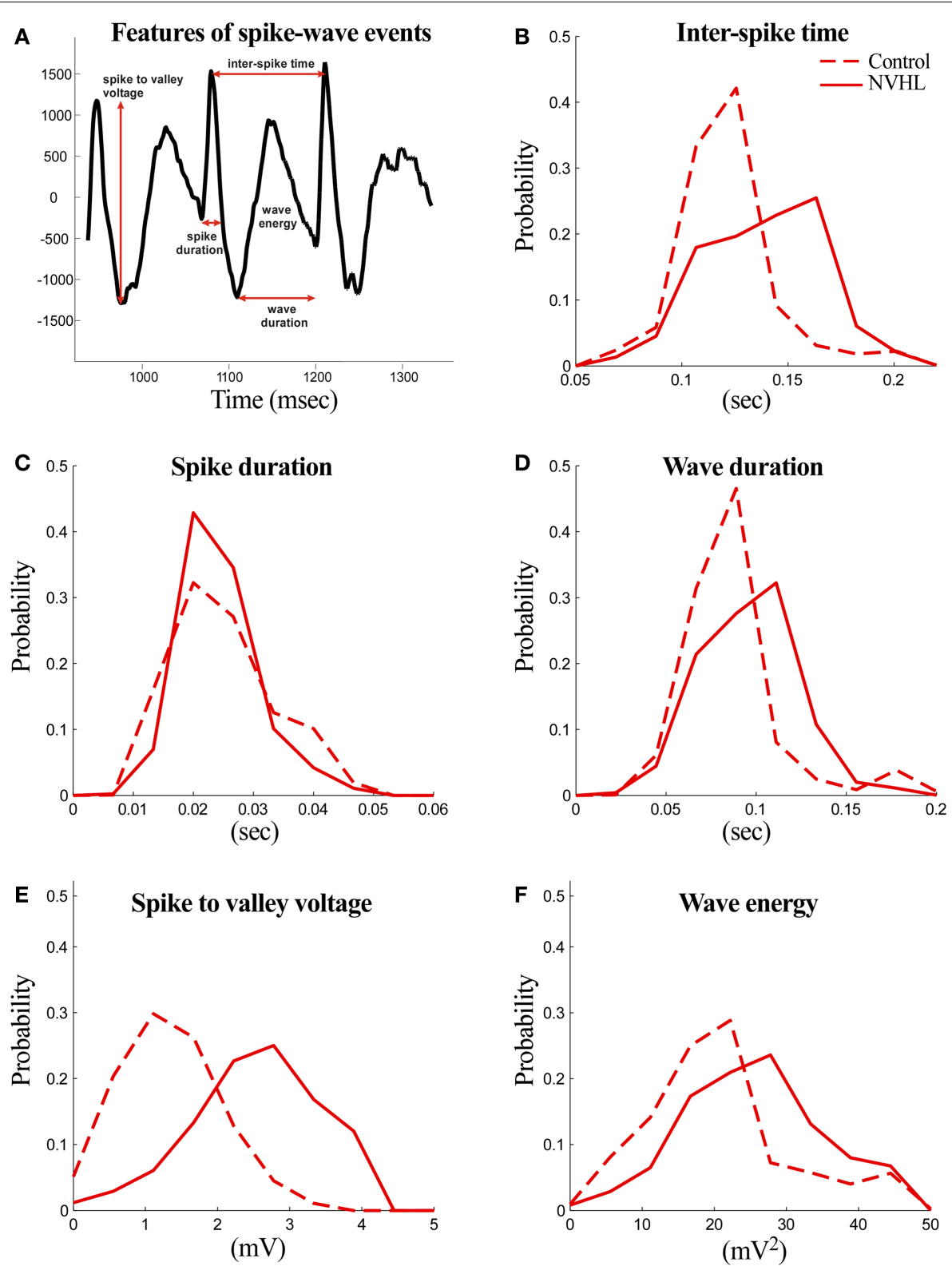

FIGURE 3 | Abnormal features of the spike-wave activities in NVHL rats (A) Three spike-wave cycles are shown to illustrate the features that were measured. The analyzed features include spike duration, inter-spike time, wave duration, spike-to-valley voltage, and inter-spike time. Comparison of the spike-wave complex features in NVHL and control rats: (B) inter-spike time,

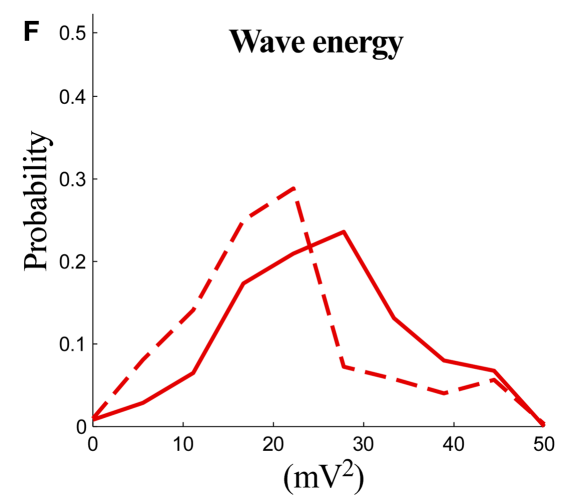

(C) spike duration, (D) wave duration, (E) spike-to-valley voltage, and (F) wave energy. Distributions in each feature looked distinct, except in spike duration, and this was confirmed statistically by the two-sample Kolmogorov-Smirnov test in which the null hypothesis that the control and NVHL distributions were drawn from the same underlying distribution was rejected $(p<0.05)$.

these events and will call them spike-wave activity. They typically occurred in trains of $\sim 6 \mathrm{~Hz}$ (Figure 2A). These events have been observed in the cortical EEG of normal Long-Evans rats during stillness and are a sign of heightened synchrony of cortical neural activity, perhaps the rat analog of the human alpha-like mu rhythm $(32,34)$. We automatically detected the individual events and confirmed by visual inspection that the algorithm properly detected the events (Figures 2B,C). Recordings from the epidural cortical screw electrodes were used for analysis because signals from the epidural cortical electrodes showed the strongest spike-wave waveforms across all animals. We analyzed a total of nine NVHL and seven control rats during home-cage behavior to estimate the prevalence of the spike-wave activity. Each rat was recorded for $3-5 \mathrm{~h}$ in the home-cage. The spike-wave activity was detected in six of nine NVHL rats and three of seven control rats. The prevalence was 3\% across all the NVHL rats and $2 \%$ in all the control rats. In total, 1514 spike-wave events were detected in NVHL rats and 397 spike-wave events were detected in control rats, suggesting that these events were more common in NVHL rats. 


\section{A}

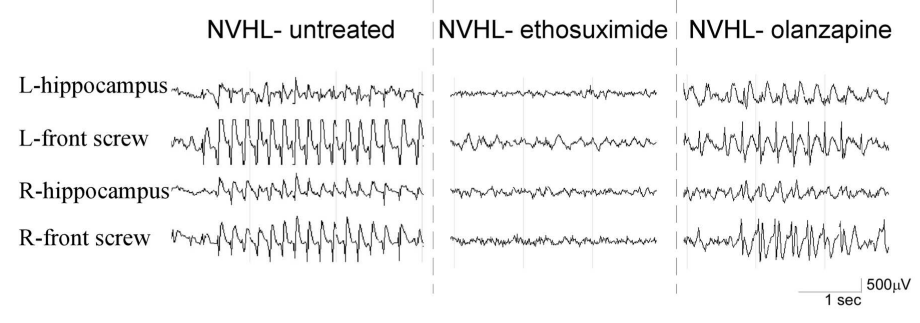

B

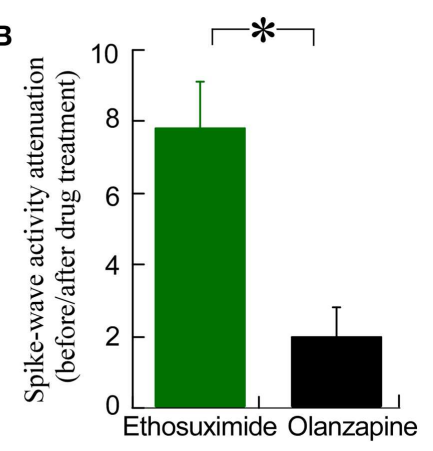

FIGURE 4 | Ethosuximide reduced the prevalence of abnormal spike-wave activities in NVHL rats. (A) Raw EEG traces.

Ethosuximide reduced the occurrence of spike-wave activity while olanzapine did not. (B) Quantification of the spike-wave activity prevalence before and after drug treatment. There was an eightfold reduction of the prevalence after ethosuximide treatment, while only a twofold attenuation of the prevalence after olanzapine treatment in NVHL rats $\left(t_{4}=3.79, p=0.02\right)$.
We then measured various features of the spike-wave events in both NVHL and control rats (Figures $\mathbf{3 A - F}$ ). The distributions of NVHL and control groups looked distinct in all the features except in spike duration. These were confirmed statistically by the two-sample Kolmogorov-Smirnov test because the null hypothesis that the control and NVHL distributions are drawn from the same underlying distribution was rejected $(p<0.05)$ for all features. These events tended to be larger amplitude and more frequent in NVHL rats in comparison to the control rats. Since we have previously characterized a cognition-related abnormality in neural synchrony in NVHL rats, in the framework of the discoordination hypothesis, these observations of abnormal synchrony suggested to us that reducing the spike-wave activities could improve cognition and related neural activity in NVHL rats.

\section{ETHOSUXIMIDE REDUCED THE PREVALENCE OF ABNORMAL SPIKE-WAVE ACTIVITIES}

We then evaluated whether the spike-wave activities could be attenuated by ethosuximide, an anticonvulsant that is an antagonist of T-type calcium channels (35). It is used mainly to treat absence seizures. Given the framework that animal models are best utilized to test hypotheses about schizophrenia and that it is unwise to assume that models themselves mimic the disease (36), we reasoned that since ethosuximide is effective at suppressing epileptiform SWDs, then it may be also be effective on the spike-wave activities in the NVHL rats. Since it might suppress this specific form of abnormal neural synchrony by rebalancing excitation and inhibition, it occurred to us that ethosuximide could also act to reduce the cognition-related abnormal neural synchrony, which according to the discoordination hypothesis would attenuate the cognitive deficit observed in these rats.

For comparison, we also chose to study the effects of olanzapine, a compound with antagonist actions at dopaminergic, muscarinic, adrenergic, GABAergic, and histaminergic receptors. It is one of the most widely used second-generation antipsychotic drugs. These drugs were designed to suppress hyperlocomotion in animal models and are prescribed to reduce the positive symptoms of psychosis, but are not considered to be effective on cognitive symptoms.
We observed that ethosuximide reduced the spike-wave activity much more than olanzapine (Figure 4A). There was an eightfold reduction in the prevalence of spike-wave activity after ethosuximide $(100 \mathrm{mg} / \mathrm{kg}$ i.p.) treatment, while only a twofold reduction in spike-wave activity in the NVHL rats (Figure 4B) after a dose of olanzapine ( $1 \mathrm{mg} / \mathrm{kg}$ i.p.) that is sufficient to attenuate sensorimotor deficits such as hyperlocomotion in the NVHL (see Figure 7C below) and acute PCP animal models (37). These observations motivated us to test whether ethosuximide can attenuate the abnormal cognition-related neural synchrony that we have previously characterized in NVHL rats (12).

\section{ETHOSUXIMIDE ATTENUATED ABNORMAL INTER-HIPPOCAMPAL SYNCHRONY}

We then turned to the main effort of this research. We recorded LFPs while the rats performed the two-frame active place avoidance task. We previously identified that oscillations in the theta and beta ranges was less synchronized between the left and right dorsal hippocampi in NVHL rats than in control rats and that this inter-hippocampal synchrony was correlated with effective place avoidance behavior (12). Inter-hippocampal theta and beta synchrony was weaker in the NVHL rats compared to the controls (Figure 5A), which was already shown in prior work (12). We administered ethosuximide and olanzapine just prior to the first place avoidance training trials in new NVHL and control groups. The difference in inter-hippocampal synchrony between the NVHL and control rats was attenuated by ethosuximide (Figure 5B), suggesting that ethosuximide normalized interhippocampal synchrony in NVHL rats. Following olanzapine, inter-hippocampal synchrony was different between the NVHL and control groups (Figure 5C). However, this difference appeared because the drug increased synchrony in the NVHL rats and decreased synchrony in the control group, especially in the theta and beta bands. Indeed, after olanzapine, synchrony in the NVHL rats was greater than in the treated control rats. Olanzapine also increased inter-hippocampal synchrony in the faster frequency gamma bands. This increase was in excess of the synchrony seen in untreated control rats, which in contrast to ethosuximide, is why olanzapine did not restore normal synchrony. 


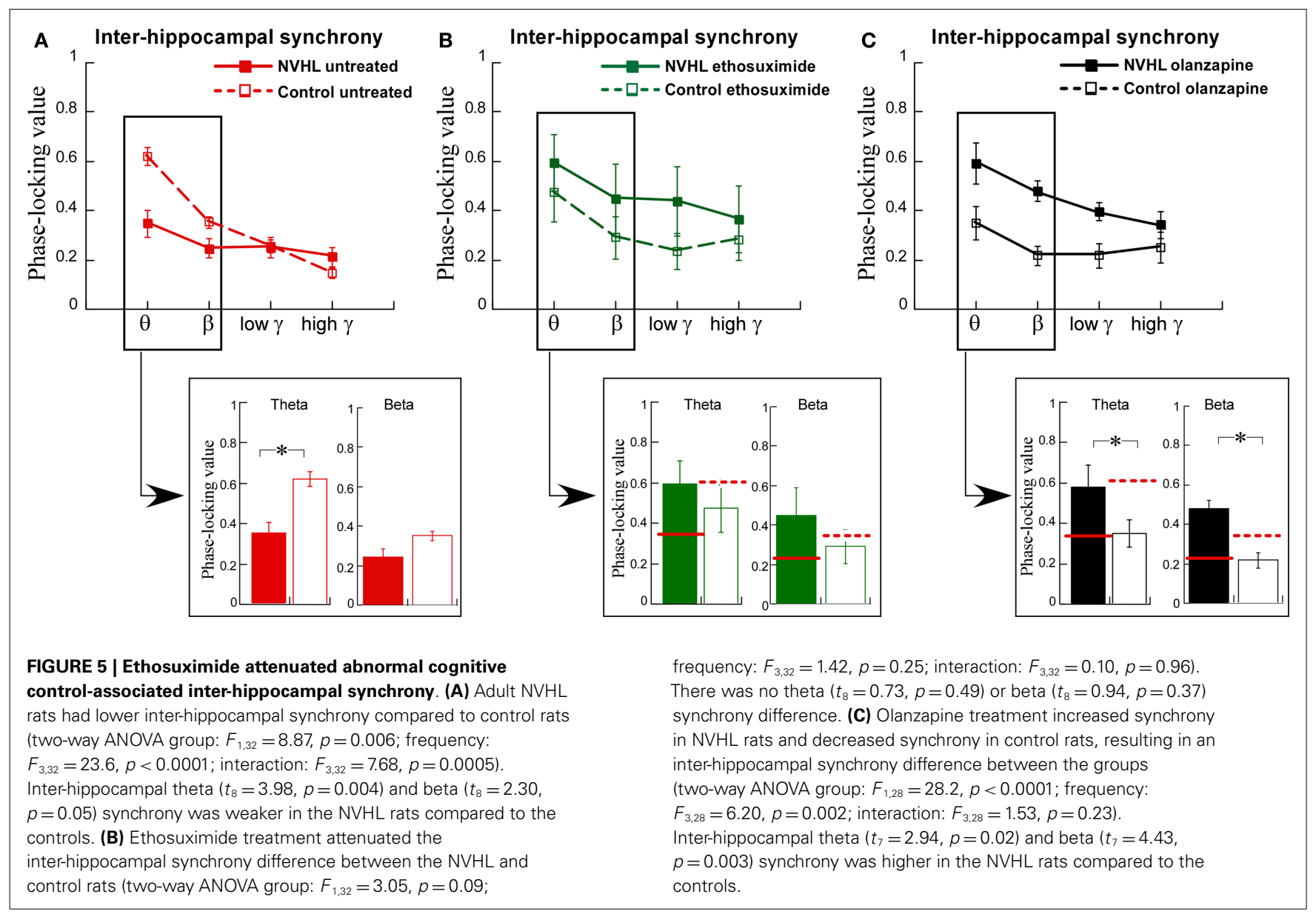

We also examined inter-hemispheric synchrony between electrodes in the mPFC (Figure 6A). The synchrony of LFP oscillations between the left and right-mPFC was greater for slower frequencies than faster frequencies but not different between the NVHL and control groups. After ethosuximide, the frequency dependence on the magnitude of synchrony between the left and right-mPFC was no longer observed. This was in large part because ethosuximide increased synchrony at the faster gamma frequencies, almost to the level of the slower beta frequencies. This effect was more pronounced in the NVHL animals. Olanzapine had a similar effect on synchrony between the left and right-mPFC sites such that there was no longer a detectable difference in the magnitude of synchrony in the different frequency bands and the NVHL and control groups were also indistinguishable.

Finally, we examined synchrony between the mPFC and dorsal hippocampus of one hemisphere (Figure 6B). Synchrony was generally less than it was between the two hippocampi or between the two mPFC. There was greater synchrony in slower frequency bands than the faster gamma bands. There was, however, no difference between NVHL and control animals. After ethosuximide, synchrony increased, especially in the faster bands, for both the NVHL and control animals. This removed the effect that synchrony was greater at slow frequencies. The NVHL and control groups were also indistinguishable after ethosuximide. Olanzapine increased synchrony in both NVHL and control rats but the increase was greater for the NVHL rats. Synchrony increased in all bands so there was no effect of the frequency band. Clearly, both ethosuximide and olanzapine altered the synchrony of oscillations in the LFPs of control and NVHL rats.

\section{ETHOSUXIMIDE ATTENUATED THE COGNITIVE CONTROL IMPAIRMENT OF NVHL RATS}

We examined whether these changes in synchrony were associated with changes in behavior using the active place avoidance task variant that allows evaluation of the ability to use relevant information and ignore irrelevant information, what is known as cognitive control. We first examined the effects of the drugs on locomotor hyperactivity (Figure 7A), which is a feature of NVHL rats that we have previously observed during the place avoidance task (12). Indeed, hyperactivity has been widely used to model the positive symptoms of schizophrenia and the effect of olanzapine in reducing hyperactivity has been shown in a variety of schizophrenia-related animal models $(38,39)$. Ethosuximide significantly reduced hyperactivity in NVHL rats compared to control rats (Figure 7B). As expected, olanzapine also reduced hyperactivity in NVHL rats compared to controls rats, so that the two groups were indistinguishable (Figure 7C). 

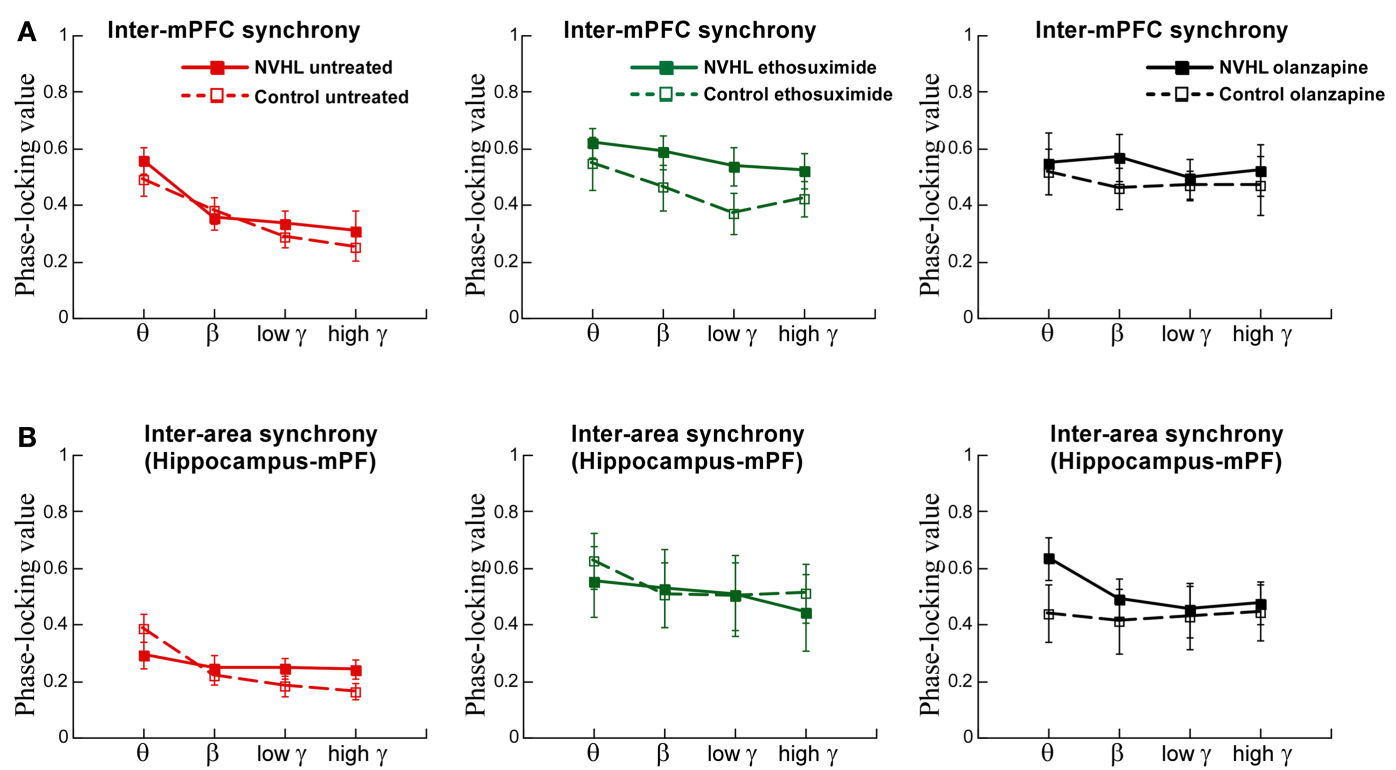

FIGURE 6 | Inter-mPFC or inter-hippocampus-mPFC phase synchrony was not different in the NVHL and control groups during active place avoidance. (A) Inter-mPFC synchrony was frequency-dependent but not different between the NVHL and control rats in the untreated groups (two-way ANOVA group: $F_{1,32}=0.92, p=0.34$; frequency: $F_{3,32}=8.97$, $p=0.0001$; interaction: $F_{3,32}=0.33, p=0.81$ ). The inter-mPFC synchrony was greater in the NVHL rats in the ethosuximide-treated group and the frequency dependence was lost due to increased synchrony at the gamma frequencies (middle: two-way ANOVA group: $F_{1,32}=5.42, p=0.03$; frequency: $F_{3,32}=1.41$, $p=0.26$; interaction: $F_{3,32}=0.16, p=0.92$ ). In the olanzapine-treated rats, the group and frequency dependencies were no longer observed because the drug increased synchrony at all frequencies we assessed (right: two-way ANOVA group: $F_{1,28}=1.43, p=0.24$; frequency: $F_{3,28}=0.40, p=0.76$; interaction: $F_{3,28}=0.11, p=0.95$ ) groups. (B) Inter-area synchrony (between $\mathrm{mPFC}$ and hippocampus) was frequency-dependent but not different between the NVHL and control rats in the untreated group (left: two-way ANOVA group: $F_{1,32}=0.43, p=0.51$; frequency: $F_{3,32}=5.29, p=0.005$; interaction: $F_{3,32}=2.05, p=0.13$ ). There were no group or frequency dependencies in the ethosuximide-treated rats because the drug increased synchrony at all frequencies to the level of the maximal (theta) inter-hippocampal synchrony (middle: two-way ANOVA group: $F_{1,32}=0.11, p=0.74$; frequency: $F_{3,32}=0.30$, $p=0.83$; interaction: $F_{3,32}=0.07, p=0.97$ ). The olanzapine-treated NVHL rats had greater synchrony at all frequencies and there was no frequency dependence because the drug also increased synchrony at all frequencies (right: two-way ANOVA group: $F_{1,28}=1.22, p=0.28$; frequency: $F_{3,28}=0.26$, $p=0.85$; interaction: $F_{3,28}=0.19, p=0.90$ ) groups.
We then examined the effects on cognitive behavior. NVHL rats were slower to learn the place avoidance (Figure $\mathbf{8 A}$ ), as we have previously reported (12). Ethosuximide attenuated the deficit because under ethosuximide NVHL rats performed at a level that was statistically indistinguishable from control rats, although there was a trend for them to be slower to learn in the initial trials (Figure 8B). The olanzapine treatment did not improve the cognitive performance of NVHL rats, which remained impaired compared to control rats (Figure 8C). In fact, olanzapine seemed to slightly impair avoidance of the control rats during the initial trials. This was confirmed by comparing the three groups of control rats from Figure 7 by two-way ANOVA (treatment: $F_{2,96}=9.70, p=0.0001$; trials: $F_{7,96}=33.86, p<0.0001$; interaction: $F_{14,96}=1.24, p=0.26$; post hoc tests: only olanzapine-treated control rats differed from ethosuximide-treated and untreated control rats).

Next we investigated the relationship between place avoidance performance and inter-hippocampal synchrony in the theta and beta bands. In the untreated NVHL and control rats, greater phaselocking at theta and beta frequencies was significantly correlated $(r$ 's $>0.8 ; p$ 's $<0.01)$ with better place avoidance measured as lower total entrances (Figure 9A) but not with hyperactivity, measured as running speed (Figure 9B). The relationship between higher synchrony and lower entrances was apparent but not significant in the ethosuximide-treated animals (theta: $r=0.37$; $p>0.1$; beta: $r=0.14 ; p>0.1$ ). Because performance was good in most of these rats, there was limited variability in behavior for the synchrony measures to explain. The trend of a relationship between synchrony and cognition was reversed in the olanzapinetreated rats. Greater synchrony was associated with worse performance, but this trend was not significant (theta: $r=0.50 ; p>0.1$; beta: $r=0.35 ; p>0.1$ ).

Finally, in the effort to describe neural coordination during the place avoidance task, we examined the full set of 32 synchrony measures ( 8 electrode pairs $\times 4$ frequency bands) as a pattern to investigate if there were group specific patterns of synchrony (Figure 10). The 32-dimensional space defined by the synchrony vectors (Figure 10A) was reduced to a 3-dimensional space to visualize the data using the first three principal components (Figure 10B). The data formed several clusters instead of filling the parameter space, which suggests neural activity may preferentially occupy preferred locales of this parameter state space. The untreated NVHL and control animals appeared to form two clusters. The ethosuximide and olanzapine-treated groups occupied distinct regions of the space away from the untreated groups. To estimate how distinct the groups might be, we computed the average distance amongst all pairs of vectors within a treatment group. The first six principal components accounted 

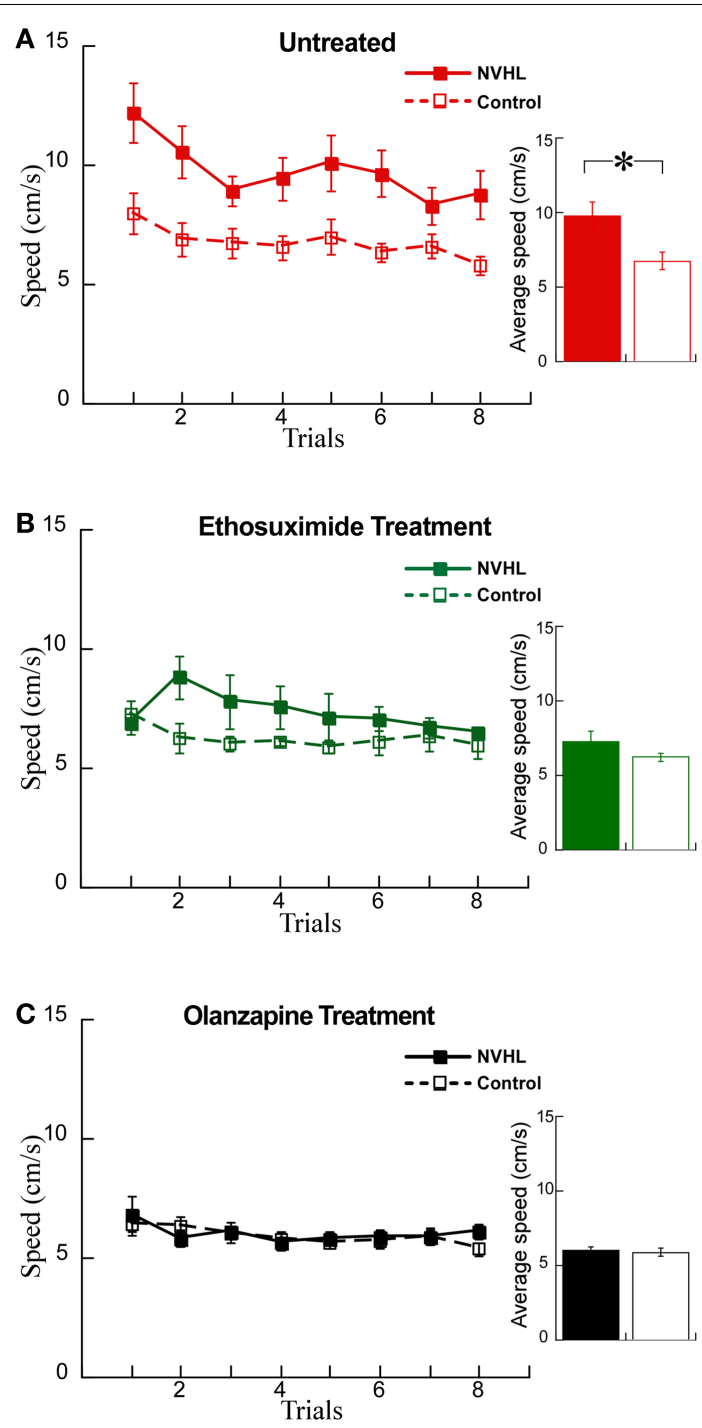

FIGURE 7 | Olanzapine and ethosuximide reduced hyperactivity in NVHL rats. (A) In the untreated group, the NVHL rats were hyperactive compared to control rats while performing the two-frame avoidance task (performance curve: two-way repeated-measures ANOVA group: $F_{1,64}=53.34, p<0.0001$; trial: $F_{7,64}=2.37, p=0.03$; interaction: $F_{7,64}=0.46$, $p=0.86)$; inset: average speed: $t_{8}=2.78, p=0.02$ ). (B) Ethosuximide treatment reduced hyperactivity in the $\mathrm{NVHL}$ rats compared to untreated NVHL rats, and showed a statistical difference when compared to control rats (performance curve: two-way repeated-measures ANOVA group: $F_{1,64}=11.68, p=0.001$; trial: $F_{7,64}=0.86, p=0.54$; interaction: $F_{7,64}=1.09$, $p=0.38)$; inset: average speed: $t_{8}=1.5, p=0.17$ ). (C) Olanzapine treatment reduced hyperactivity so there was no longer a difference from control rats (performance curve: two-way repeated-measures ANOVA group: $F_{1,64}=0.36, p=0.55$; trial: $F_{7,64}=1.37, p=0.23$; interaction: $\left.F_{7,64}=0.48, p=0.85\right)$; inset: average speed: $\left.t_{8}=0.30, p=0.77\right)$.

for over $90 \%$ of the variance in the data set (Figure 10C), so we computed the pair-wise distances as the 6-D Euclidean distance. The average distance from the untreated NVHL group was significantly greater for each group than it was for the vectors in the untreated NVHL group (Figure 10D). Similarly, the
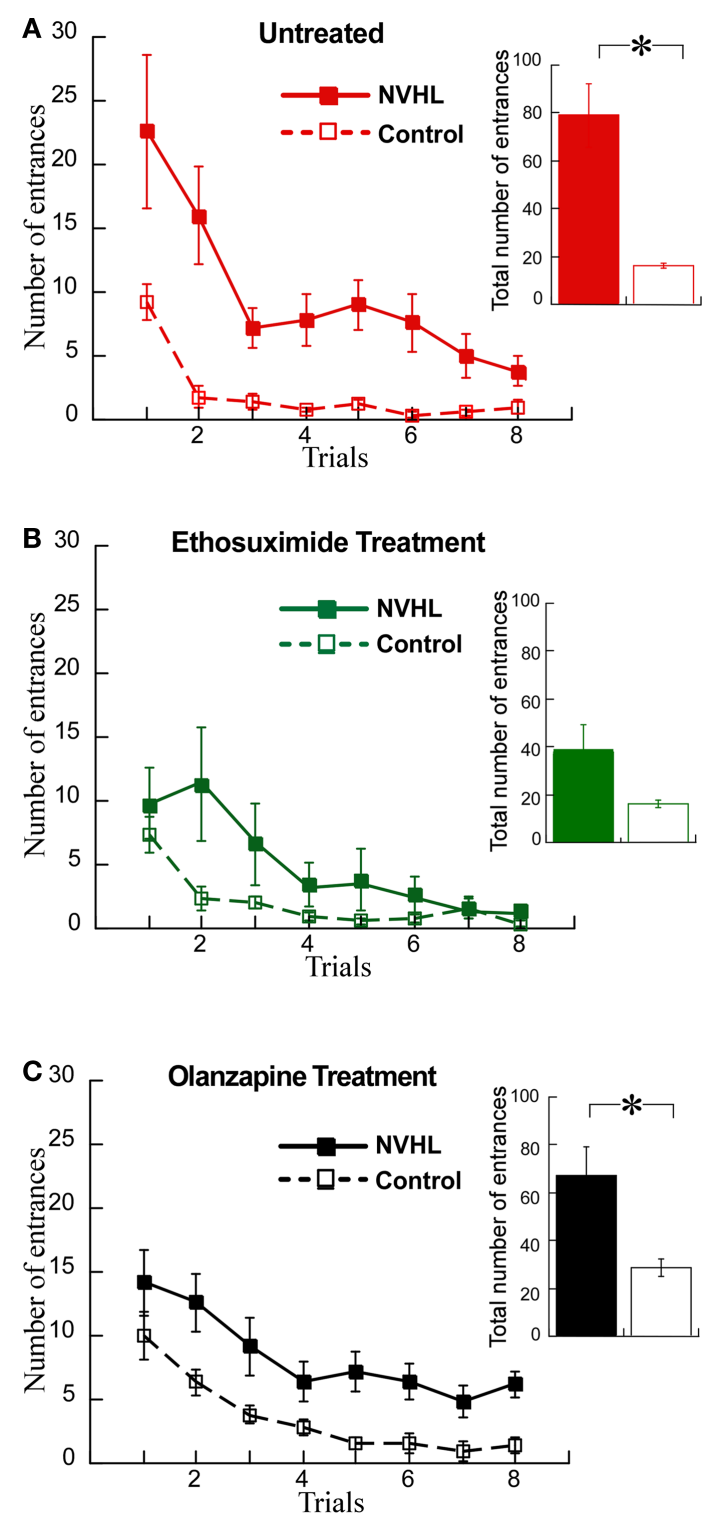

FIGURE 8 | Ethosuximide attenuated the cognitive control impairment of NVHL rats. (A) In the untreated group, the NVHL rats were slower to learn the two-frame task and showed an impairment when compared to the control rats (learning curve: two-way repeated-measures ANOVA group: $F_{1,64}=53.84, p<0.0001$; trial: $F_{7,64}=8.80, p<0.0001$; interaction: $\left.F_{7,64}=1.79, p=0.10\right)$; inset: total number of entrances: $t_{8}=4.69$, $p=0.002$ ). (B) Ethosuximide treatment attenuated the learning impairment in the NVHL rats when compared to control rats (learning curve: two-way repeated-measures ANOVA group: $F_{1,64}=5.86, p=0.02$; trial: $F_{7,64}=9.79$, $p<0.0001$; interaction: $\left.F_{7,64}=2.40, p=0.03\right)$; inset: total number of entrances: $\left.t_{8}=1.87, p=0.10\right)$. (C) Olanzapine treatment did not improve performance of the NVHL rats on the two-frame task (learning curve: two-way repeated-measures ANOVA group: $F_{1,64}=42.87, p<0.0001$; trial: $F_{7,64}=9.66, p<0.0001$; interaction: $\left.F_{7,64}=0.19, p=0.99\right)$; inset: total number of entrances: $t_{8}=3.10, p=0.02$ ).

distances from the untreated control vectors were significantly greater for all other groups except the control group treated with ethosuximide, suggesting that ethosuximide did not significantly 


\section{A Inter-hippocampal synchrony is correlated with place avoidance performance in Untreated groups}
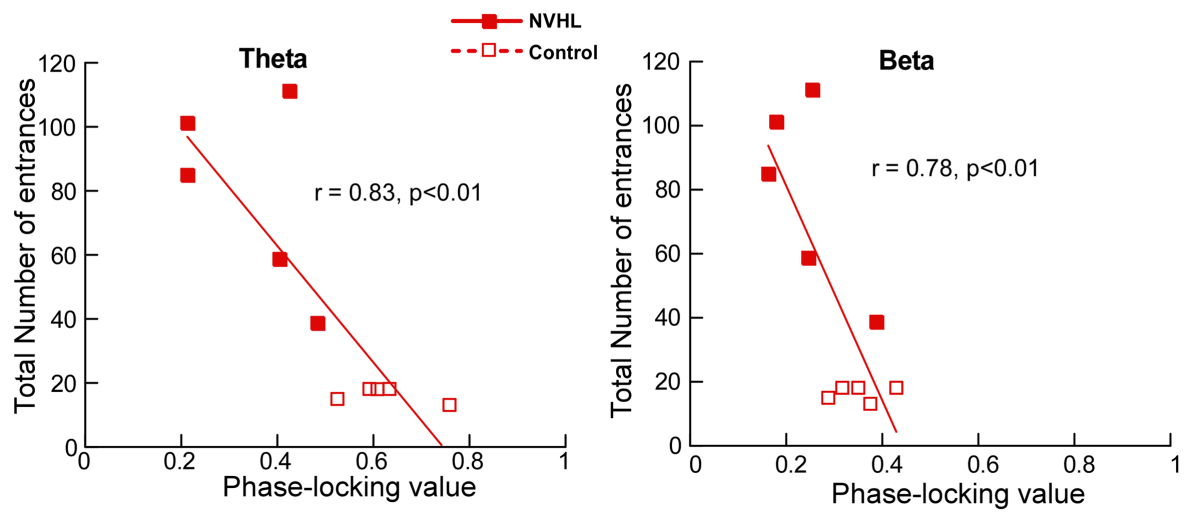

B Inter-hippocampal synchrony is not correlated with hyperactivity in Untreated groups

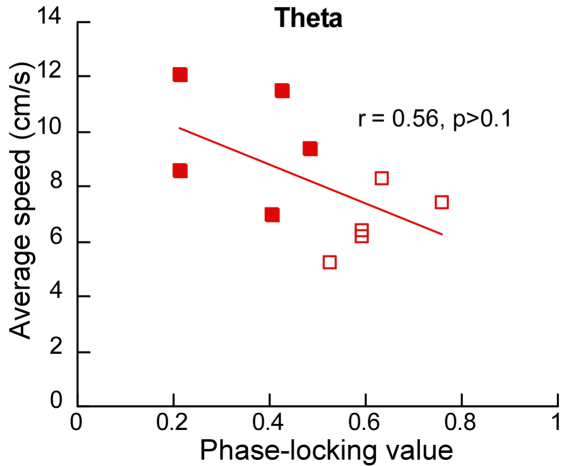

FIGURE 9 | Inter-hippocampal synchrony is correlated with place avoidance performance. (A) In the untreated NVHL and control rats, the scatter plot distributions of theta phase-locking values (left) and beta

phase-locking values (right) show significant correlation with the total number

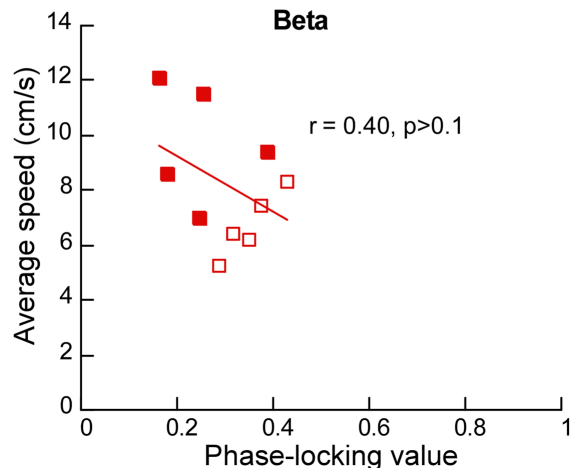

of errors in the two-frame avoidance task. (B) In the untreated NVHL and control rats, theta phase-locking values (left) and beta phase-locking values (right) were not correlated with hyperactivity, measured as running speed during the two-frame avoidance task. change control synchrony patterns. Because place avoidance behavior in the NVHL group was improved by ethosuximide, we estimated the distance between this group and the others. The distance to the untreated control group was no different than the distance within the NVHL-ethosuximide group, suggesting that the two groups were relatively similar. The complementary estimate of distance, this time to the olanzapine-NVHL group had a different answer. All groups had greater distances to the olanzapine-NVHL group than the distances amongst the group itself, consistent with the synchrony pattern caused by olanzapine being unique, rather than normalizing.

\section{DISCUSSION}

\section{SUMMARY}

The main finding of this study is that a drug that normalizes aberrant cognition-related neural synchrony in NVHL rats can also attenuate the cognitive control impairment in this schizophreniarelated neurodevelopmental model of mental dysfunction. This work demonstrates that the pattern of abnormal cognition-related neural synchrony is acutely normalized by ethosuximide, and furthermore shows that the synchrony alterations were associated with improved cognitive performance after ethosuximide but not olanzapine, despite both drugs being effective at reducing hyperactive locomotion in the NVHL model. Although olanzapine increased theta and beta synchrony between the two hippocampi, which is correlated with task performance (Figure 8), the drug also increased gamma synchrony beyond baseline levels, which may itself be associated with poor place avoidance performance in mutant mice $(40,41)$. Note that under ethosuximide, and also under olanzapine, the overall neural synchrony pattern in NVHL rats was distinct from the untreated control pattern, but the pattern after ethosuximide was closer to the control pattern than the pattern after olanzapine (Figure 10D) and perhaps this is one of the reasons that place avoidance performance was improved by ethosuximide but not olanzapine.

We have used the NVHL model to examine abnormal synchrony and cognitive impairment. Although NVHL rats are not a model of schizophrenia per se, these animals are an experimental 

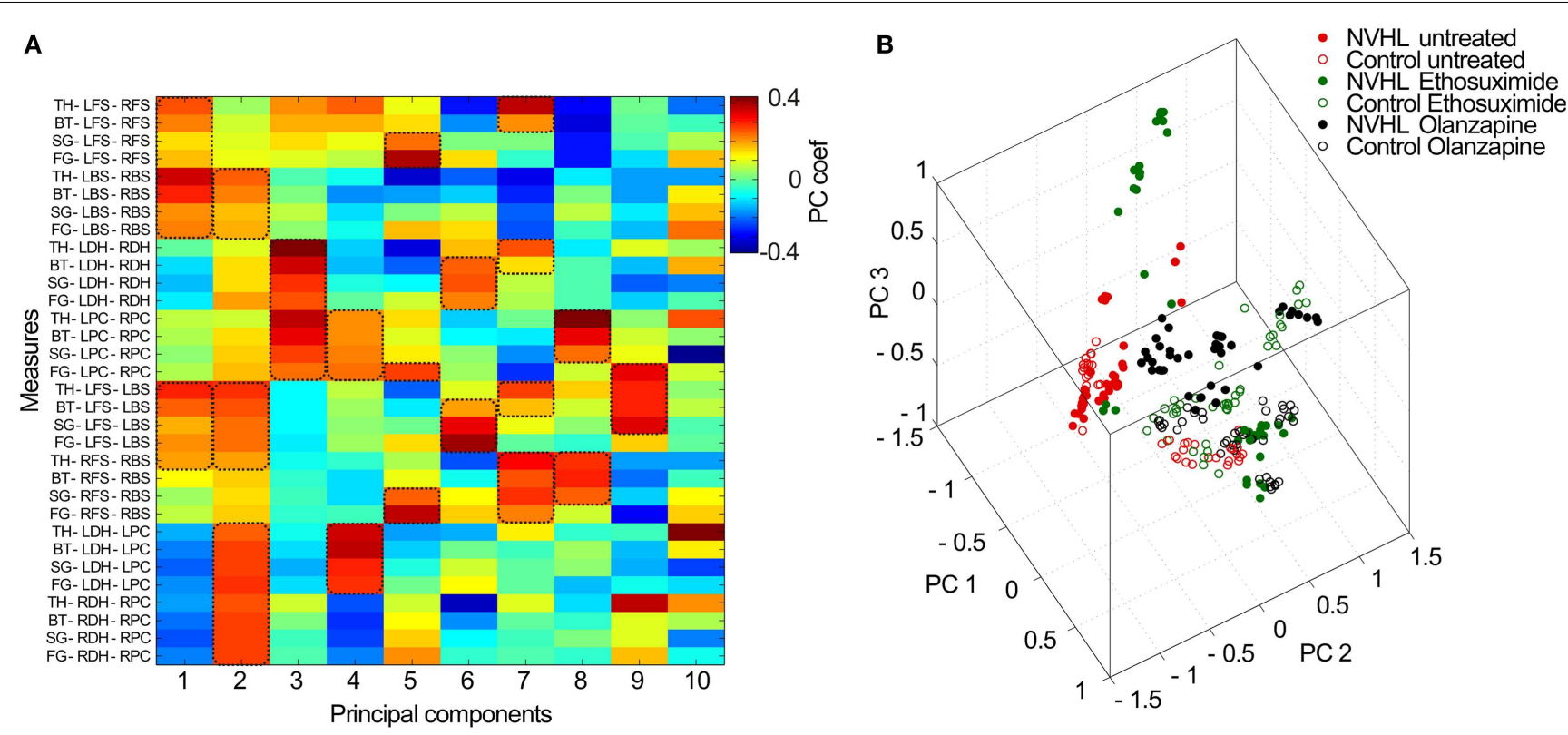

C

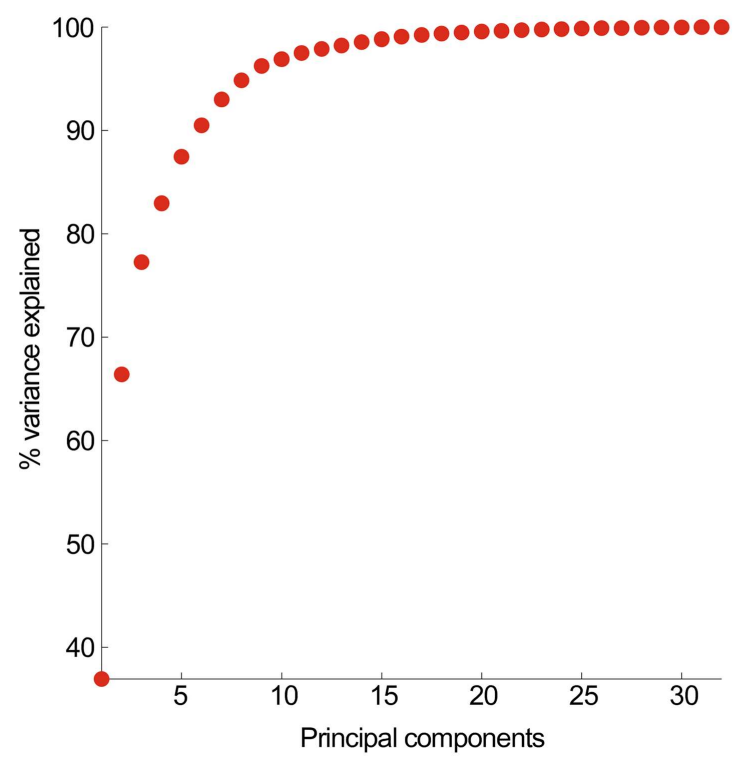

D Distance from NVHL - untreated $* \mathrm{p}<0.0025$
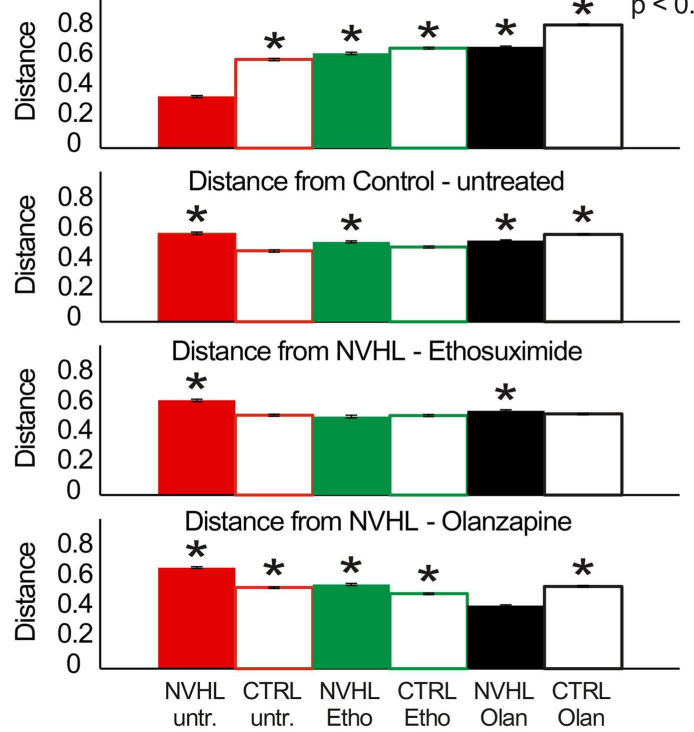

FIGURE 10 | Analysis of the multidimensional pattern of synchrony. (A) Thirty-two PLV measures were extracted from the data (8 electrode pairs $\times 4$ frequency bands) and treated as a set of 32-dimensional vectors. PCA analysis was performed on the resulting vector dataset. The first 10 principal components $(\mathrm{PC})$ are shown here ( $x$-axis) with the relative contribution of each measurement ( $y$-axis) indicated by a color code. The PCs tend to group measures with similar properties. As an example, PC \#1 corresponds mostly to frequency band-non-specific phase synchrony between the contralateral cortical electrode pairs as well as ipsilateral cortical electrode pairs, which are measures of global cortical phase synchrony. PC \#3 corresponds mostly to frequency band-non-specific phase synchrony between the left and right-hippocampi as well as between the left and right prefrontal cortex, which is an estimate of region-specific inter-hemispheric synchrony. PC \#6 corresponds to frequency-specific synchrony in the beta,

slow gamma, and fast gamma bands between inter-hemispheric neocortical and hippocampal sites. The key measures of synchrony that contributed to each PC are indicated by the dashed boxes. (B) The multidimensional data can be visualized by plotting in the 3-D space of the first three PCs. (C) The cumulative probability of the dataset variance is explained by the PCs. The first six principal components explain $\sim 90 \%$ of the dataset variance. (D) The average Euclidean distance between a pair of vectors estimates their distinctiveness. The distances between all pairs of vectors were computed within a treatment group and between all treatment groups and a specific treatment group to estimate similarity/distinctiveness of the treatment groups. The average distances are given to the untreated NVHL group (top row), to the untreated control group (second row), to the ethosuximide-treated NVHL group (third row), and to the olanzapine-treated NVHL group (bottom row). 
model with neural synchrony and related cognitive abnormalities, which allows a prediction of the discoordination hypothesis to be tested. This unifying hypothesis was proposed to account for the schizophrenia syndrome independent of etiology $(1,2,12,27,28)$. This view acknowledges that schizophrenia may turn out to be heterogeneous and that multiple factors contribute, including genetic alterations, infectious, toxic, and stressful events $(42,43)$. Whatever the etiology, the discoordination hypothesis asserts that disruption of the physiological synchronization of neural activities produces cognitive abnormalities in schizophrenia, despite otherwise seemingly normal expression of unitary physiological phenomena. The hypothesis is based specifically on the physiological coordination of neural activities, defined as the set of neural processes that control the timing of spike discharge across ensembles of neurons $(1,29,44,45)$. Our group has demonstrated this neural coordination during cognitive control in active place avoidance tasks $(46,47)$. The current findings support the discoordination hypothesis by demonstrating that correcting the neural synchrony abnormality in NVHL rats can be associated with cognitive improvement, which was the case with ethosuximide but not with olanzapine. Olanzapine did not normalize either neural synchrony or cognitive behavior (Figures 4, 5, and 8). In further support of the hypothesis, olanzapine caused abnormal neural synchrony in control rats, which seemed to worsen the place avoidance.

\section{SPIKE-WAVE ACTIVITY}

A secondary finding in this study is that spike-wave like activity was observed in the epidural cortical EEG of both NVHL and control rats during stillness in the home-cage and only rarely during active place avoidance behavior, consistent with prior observations $(32,34)$. This observation may have implications for how deficits in NVHL animals are interpreted when those deficits are characterized in tasks during which there is behavioral stillness, like pre-pulse inhibition, inhibitory avoidance, and food-motivated radial-arm mazes. Complex interactions of inhibitory and excitatory systems between the thalamus and cortical structures generate a variety of normal and abnormal rhythmic states including spindle waves and SWDs during absence seizures. Spindle waves are characterized by $1-4$ s periods of $6-14 \mathrm{~Hz}$ oscillations during normal sleep (48). The characteristic frequency of the SWD is $3-4 \mathrm{~Hz}$ in humans (49) and is normally higher $(4-12 \mathrm{~Hz})$ in rodents $(50,51)$. Our analysis of cortical spike-wave activity shows that the features of the spike-wave activity in NVHL and control rats are different (Figure 2). The duration of the activity was greater in NVHL rats compared to control rats, further suggesting that this activity in the NVHL rats may be of a different neural origin than it is in control rats. In NVHL rats it may reflect a form of pathological network synchrony and even a sign of epileptiform propensity (52).

Indeed, during several hours of continuous monitoring, we have observed rare, but nonetheless bona fide epileptiform discharges and behavioral seizures in NVHL rats (52). The NHVL rats may have increased susceptibility to seizures due to dysregulation of the GABA system $(53,54)$. There is widespread reduction of GAD67 expression $(54,55)$, and decreased expression of PV interneurons in the prefrontal cortex and the hippocampus (54) in these rats. There is also increased firing in pyramidal neurons in the NVHL rats. The mPFC pyramidal neurons of NVHL rats respond to stimulation of the ventral tegmental area, the origin of dopamine projections to the PFC, with an increase in firing rates (56) instead of the normal decrease (57), indicating hyperexcitability. It has been shown that NVHL rats developed epileptiform SWDs at a twofold lower dose of pentylenetetrazol (PTZ, a $\mathrm{GABA}_{\mathrm{A}}$ antagonist) than sham-operated rats, further evidence that the NVHL rats have increased susceptibility to absence-like seizures (54). Indeed, PD7 injections into ventral hippocampus with kainate, an excitotoxin with a different mechanism of action than ibotenate (58), is used as a model of temporal lobe epilepsy (59). Perturbations of the GABA system, together with the aberrant response reflecting the inability of dopamine to activate interneurons, may result in enhanced cortical excitability and abnormal spike-wave activity in NVHL rats.

\section{ETHOSUXIMIDE TREATMENT}

We now discuss the effects of ethosuximide in the contexts of its pharmacological actions, typical use as well as the relevance of the findings with ethosuximide to understanding the cognitive impairments in the NVHL rat model and mental illness such as schizophrenia. To be clear, neither the findings nor the discussion that follows suggest that ethosuximide might be an effective treatment for schizophrenia. Rather, the procognitive and normalizing neural coordination effects of ethosuximide in the NVHL model provides new evidence that attenuating neural synchrony deficits can itself be procognitive.

Ethosuximide is used extensively to treat absence (petit mal) seizures (60-62). The mechanism of action is to block lowthreshold $\mathrm{Ca}^{2+}$ currents in the T-type $\mathrm{Ca}^{2+}$ channels and enhance GABAergic tone $(63,64)$. We observed that ethosuximide reduced spike-wave activity in NVHL rats, indicating it is effective on abnormal synchrony in NVHL rats during home-cage behaviors (Figure 3). The drug also increased inter-hippocampal synchrony in NVHL rats so that neural coordination between the two hippocampi approached normal levels in the theta- and betafrequency bands. Nonetheless, taken together, after ethosuximide, the overall pattern of neural synchrony in the NVHL rats was modified but the pattern of synchronies was not restored to the pattern of the untreated controls. Indeed, ethosuximide also changed the synchrony pattern in the controls animals too, but these changes were not associated with cognitive disability.

The effectiveness of ethosuximide may be contemplated in relation to the fact that the synchronization of oscillations reflects the temporally precise interaction of neural activities (65). Such coordinated interactions result from an appropriate balance between GABA-mediated inhibition and glutamate-mediated excitation, which may be unbalanced in adult NVHL rats (56). Alterations in one or both of these systems can result in abnormal network synchrony. Schizophrenia has been associated with dysregulation of cortical GABAergic neurotransmission (66) and abnormalities in NMDA-receptor mediated neurotransmission (67). There are significantly decreased levels of GABAergic neuronal markers like parvalbumin mRNA in the prefrontal cortex $(68,69)$ and in the dorsolateral prefrontal cortex of schizophrenia patients, decreased levels have been reported of glutamic acid decarboxylase (GAD67) mRNA, the GABA-synthesizing enzyme (70,71). Indeed, 
NMDA-receptor hypofunction in schizophrenia $(72,73)$ is now thought to primarily affect GABA inhibition by reducing GABAergic tone. Thus dysregulation of excitatory and inhibitory neurotransmission contribute to abnormalities in the coordination of neural activities, which according to the hypothesis, is the basis for the impaired cognitive functions that are central to schizophrenia and a logical target for developing cognition promoting therapies. It was recently proposed that aberrant low frequency delta/theta oscillations emerge as a consequence of NMDA-receptor hypofunction $(3,74)$. The reduced excitation would deinactivate T-type $\mathrm{Ca}^{2+}$ channels and exaggerate bursting, especially in thalamic neurons, which may be the origin of the low frequency thalamocortical oscillations that could disrupt cortical function in schizophrenia (75). In this context, given ethosuximide's mechanism of action, it is perhaps not surprising that the drug both normalized neural synchrony and improved cognition in NVHL rats. Ethosuximide reduces excitability primarily by blocking activity-dependent lowthreshold $\mathrm{Ca}^{2+}$ currents as well as by acting as a partial agonist at the picrotoxin GABA-blocking receptor (64).

In the absence of the forgoing discussion, it may seem surprising to some readers that ethosuximide can improve interhippocampal synchrony and place avoidance behavior. Some readers may have assumed that because ethosuximide is antiepileptic it must decrease neural synchrony because epileptiform activity is thought of as excessive synchrony. According to these opinions, ethosuximide would decrease synchrony, not increase it, and thus worsen place avoidance behavior in control as well as NVHL rats. As we have pointed out in the Section "Introduction," epileptiform activity is not simply excessive synchrony, and in the NVHL rat, we have measured the evidence of widespread decreases of synchrony between brain areas, resembling functional disconnection (52). Embedded in this loosening of neural coordination, perhaps by excitation-inhibition uncoupling, we also observed that some brain areas can exhibit relatively increased synchrony. Thus, there are alternative conceptualizations of the ethosuximide effects but they are not supported by the data on the subject in general or on NVHL rats in particular.

Ethosuximide treatment also reduced hyperactivity in NVHL rats, which in animal models, is widely studied as a behavioral analog of the positive symptoms of schizophrenia, although the reduction was not as effective as after olanzapine. Blocking T-type $\mathrm{Ca}^{2+}$ channels can produce antipsychotic effects in rats by attenuating the psychomotor effects of both the NMDA antagonist MK-801 and amphetamine, the dopaminergic psychostimulant (76). T-type $\mathrm{Ca}^{2+}$ channels are widely expressed in the brain, including areas such as the thalamus, the prefrontal cortex, the hippocampus, and the nucleus accumbens (77), which are regions that have been reported to function abnormally in schizophrenia $(74,78,79)$. Interestingly, a number of clinically validated antipsychotics, including haloperidol, pimozide, flunarizine, and clozapine, are potent T-type channel antagonists (76, 80-82). A recent genome-wide study of single-nucleotide polymorphisms (SNP) found that variations in calcium channel genes are associated with schizophrenia as well as a range of major psychiatric disorders (83). These data implicate calcium channels in the pathophysiology of schizophrenia, and while calcium channel abnormality may help to account for why ethosuximide was effective on NVHL rats, it is also possible that ethosuximide was effective because of its fundamental effects on excitation-inhibition balance through the action on otherwise normal calcium channels. Nonetheless, the present work does not imply and we do not propose to use ethosuximide as a treatment for cognitive deficits in schizophrenia. Rather the present findings demonstrate that procognitive effects may be possible if treatments can normalize the coordination of neural activity between cognitive processing centers like the two hippocampi.

\section{OLANZAPINE TREATMENT}

Second-generation (atypical) antipsychotic medications, including olanzapine, are effective in treating psychosis, hallucinations, and delusions (84) but ineffective in treating the cognitive impairments that strongly debilitate schizophrenia patients (85). Olanzapine, a thienobenzodiazepine derivative, is classified as a multi-acting receptor-targeted antipsychotic, showing high affinity for dopaminergic, serotonergic, cholinergic, histaminergic, and muscarinic receptors $(86,87)$. As expected, we observed that olanzapine reduced hyperactivity in NVHL rats to control levels during the place avoidance task, indicating we used an effective antipsychotic dose, at least by traditional measures. The dose was however, ineffective at improving cognitive performance of NVHL rats in the two-frame task. This is of course consistent with the drug's lack of effect on cognitive symptoms in patients.

Olanzapine treatment in NVHL and control rats showed a reversed effect on neural synchrony. Olanzapine increased interhippocampal synchrony in NVHL rats to the level of untreated controls in the theta and beta bands and in excess of the control levels in the gamma bands. In contrast, the drug decreased inter-hippocampal synchrony in control rats. The increased synchrony in olanzapine-treated NVHL rats was not associated with improved cognitive performance in the NVHL rats, which would be at odds with the discoordination hypothesis, except that after olanzapine, the gamma synchrony was excessive, indicating that olanzapine changed neural coordination, but as demonstrated with the multidimensional synchrony analysis (Figure 10), the synchrony changes did not normalize neural synchrony in NVHL rats. Conversely, but also in support of the discoordination hypothesis, the decreased synchrony in olanzapine-treated control rats was associated with a higher number of errors in the two-frame task when compared to untreated control rats, which is consistent with the observation that the drug changed the neural synchrony pattern in control animals to a pattern that is substantially distinct (Figures 10B,D) from the untreated control pattern. It is well documented that antipsychotic drugs cause EEG abnormalities associated with general slowing of background activity, an increase in paroxysmal theta or delta activity and the development of epileptiform discharges (88). In patients on olanzapine, there is an increased diffuse and intermittent slowing of the EEG (89, 90 ) and an increased high risk of EEG abnormalities such as theta and delta slowing, sharp waves or phase reversal, and/or spikewave activities $(88,91)$. Healthy subjects on olanzapine showed an increased power in the theta band and a decrease in the beta band (92). In summary, olanzapine normalized inter-hippocampal synchrony in the theta and beta bands but it also caused abnormal synchrony changes in both the NVHL and control rats that were not 
associated with improved cognitive performance in the two-frame task.

\section{CONCLUSION}

The findings reported here are consistent with the main assertion and prediction of the discoordination hypothesis that abnormal neural synchrony during cognitive effort is associated with impaired cognition and that restoring normal synchrony will promote cognition. As shown here, targeting the pathophysiology of abnormal neural coordination, regardless of the etiology, may be both a rational and effective program for developing a muchneeded generation of procognitive treatments for mental illness in general and schizophrenia in particular.

\section{AUTHOR CONTRIBUTIONS}

Heekyung Lee collected and analyzed data, Dino Dvorak analyzed data, Heekyung Lee and André A. Fenton wrote the manuscript, and André A. Fenton planned and organized the research.

\section{ACKNOWLEDGMENTS}

This work was supported by NIMH grant R01MH084038.

\section{REFERENCES}

1. Phillips WA, Silverstein SM. Convergence of biological and psychological perspectives on cognitive coordination in schizophrenia. Behav Brain Sci (2003) 26:65-82. doi:10.1017/S0140525X03000025

2. Uhlhaas PJ, Singer W. Neural synchrony in brain disorders: relevance for cognitive dysfunctions and pathophysiology. Neuron (2006) 52:155-68. doi:10.1016/ j.neuron.2006.09.020

3. Lisman JE, Coyle JT, Green RW, Javitt DC, Benes FM, Heckers S, et al. Circuitbased framework for understanding neurotransmitter and risk gene interactions in schizophrenia. Trends Neurosci (2008) 31(5):234-42. doi:10.1016/j.tins.2008. 02.005

4. Uhlhaas PJ, Singer W. Abnormal neural oscillations and synchrony in schizophrenia. Nat Rev Neurosci (2010) 11:100-13. doi:10.1038/nrn2774

5. Galderisi S, Mucci A, Volpe U, Boutros N. Evidence-based medicine and electrophysiology in schizophrenia. Clin EEG Neurosci (2009) 40:62-77. doi:10.1177/ 155005940904000206

6. Schmiedt C, Brand A, Hildebrandt H, Basar-Eroglu C. Event-related theta oscillations during working memory tasks in patients with schizophrenia and healthy controls. Brain Res Cogn Brain Res (2005) 25:936-47. doi:10.1016/j.cogbrainres. 2005.09.015

7. Uhlhaas PJ, Linden DE, Singer W, Haenschel C, Lindner M, Maurer K, et al. Dysfunctional long-range coordination of neural activity during Gestalt perception in schizophrenia. J Neurosci (2006) 26:8168-75. doi:10.1523/JNEUROSCI. 2002-06.2006

8. Spencer KM, Nestor PG, Perlmutter R, Niznikiewicz MA, Klump MC, Fru$\min \mathrm{M}$, et al. Neural synchrony indexes disordered perception and cognition in schizophrenia. Proc Natl Acad Sci U S A (2004) 101:17288-93. doi:10.1073/pnas. 0406074101

9. Cho RY, Konecky RO, Carter CS. Impairments in frontal cortical gamma synchrony and cognitive control in schizophrenia. Proc Natl Acad Sci U S A (2006) 103:19878-83. doi:10.1073/pnas.0609440103

10. Sigurdsson T, Stark KL, Karayiorgou M, Gogos JA, Gordon JA. Impaired hippocampal-prefrontal synchrony in a genetic mouse model of schizophrenia. Nature (2010) 464(7289):763-7. doi:10.1038/nature08855

11. Dickerson DD, Wolff AR, Bilkey DK. Abnormal long-range neural synchrony in a maternal immune activation animal model of schizophrenia. J Neurosci (2010) 30(37):12424-31. doi:10.1523/JNEUROSCI.3046-10.2010

12. Lee H, Dvorak D, Kao HY, Duffy AM, Scharfman HE, Fenton AA. Early cognitive experience prevents adult deficits in a neurodevelopmental schizophrenia model. Neuron (2012) 75:714-24. doi:10.1016/j.neuron.2012.06.016

13. Palop JJ, Chin J, Mucke L. A network dysfunction perspective on neurodegenerative diseases. Nature (2006) 443:768-73. doi:10.1038/nature05289
14. Palop JJ, Chin J, Roberson ED, Wang J, Thwin MT, Bien-Ly N, et al. Aberrant excitatory neuronal activity and compensatory remodeling of inhibitory hippocampal circuits in mouse models of Alzheimer's disease. Neuron (2007) 55:697-711. doi:10.1016/j.neuron.2007.07.025

15. Sanchez PE, Zhu L, Verret L, Vossel KA, Orr AG, Cirrito JR, et al. Levetiracetam suppresses neuronal network dysfunction and reverses synaptic and cognitive deficits in an Alzheimer's disease model. Proc Natl Acad Sci U S A (2012) 109:E2895-903. doi:10.1073/pnas.1121081109

16. Lipska BK, Jaskiw GE, Weinberger DR. Postpubertal emergence of hyperresponsiveness to stress and to amphetamine after neonatal excitotoxic hippocampal damage: a potential animal model of schizophrenia. Neuropsychopharmacology (1993) 9:67-75. doi:10.1038/npp.1993.44

17. Lawrie SM, Abukmeil SS. Brain abnormality in schizophrenia. A systematic and quantitative review of volumetric magnetic resonance imaging studies. Br J Psychiatry (1998) 172:110-20. doi:10.1192/bjp.172.2.110

18. Nelson MD, Saykin AJ, Flashman LA, Riordan HJ. Hippocampal volume reduction in schizophrenia as assessed by magnetic resonance imaging: a metaanalytic study. Arch Gen Psychiatry (1998) 55:433-40. doi:10.1001/archpsyc.55. 5.433

19. McCarley RW, Wible CG, Frumin M, Hirayasu Y, Levitt JJ, Fischer IA, et al. MRI anatomy of schizophrenia. Biol Psychiatry (1999) 45:1099-119. doi:10.1016/ S0006-3223(99)00018-9

20. Wright IC, Rabe-Hesketh S, Woodruff PW, David AS, Murray RM, Bullmore ET. Meta-analysis of regional brain volumes in schizophrenia. Am J Psychiatry (2000) 157:16-25.

21. Goldman MB, Mitchell CP. What is the functional significance of hippocampal pathology in schizophrenia? Schizophr Bull (2004) 30:367-92. doi:10.1093/ oxfordjournals.schbul.a007086

22. Harrison PJ. The hippocampus in schizophrenia: a review of the neuropathological evidence and its pathophysiological implications. Psychopharmacology (Berl) (2004) 174:151-62. doi:10.1007/s00213-003-1761-y

23. Jessen F, Scheef L, Germeshausen L, Tawo Y, Kockler M, Kuhn KU, et al. Reduced hippocampal activation during encoding and recognition of words in schizophrenia patients. Am J Psychiatry (2003) 160:1305-12. doi:10.1176/appi.ajp. 160.7.1305

24. Luck D, Danion JM, Marrer C, Pham BT, Gounot D, Foucher J. Abnormal medial temporal activity for bound information during working memory maintenance in patients with schizophrenia. Hippocampus (2010) 20:936-48. doi:10.1002/hipo.20689

25. Zierhut K, Bogerts B, Schott B, Fenker D, Walter M, Albrecht D, et al. The role of hippocampus dysfunction in deficient memory encoding and positive symptoms in schizophrenia. Psychiatry Res (2010) 183:187-94. doi:10.1016/j. pscychresns.2010.03.007

26. Erk S, Meyer-Lindenberg A, Schnell K, Opitz Von Boberfeld C, Esslinger C, Kirsch $\mathrm{P}$, et al. Brain function in carriers of a genome-wide supported bipolar disorder variant. Arch Gen Psychiatry (2010) 67:803-11. doi:10.1001/archgenpsychiatry. 2010.94

27. Fenton AA. Neural coordination and psychotic disorganization. In: Holscher C, Munk MH, editors. Information Processing by Neuronal Populations. London: Cambridge University Press (2008). p. 387-408.

28. Tononi G, Edelman GM. Schizophrenia and the mechanisms of conscious integration. Brain Res Brain Res Rev (2000) 31:391-400. doi:10.1016/S01650173(99)00056-9

29. Buzsaki G. Neural syntax: cell assemblies, synapsembles, and readers. Neuron (2010) 68:362-85. doi:10.1016/j.neuron.2010.09.023

30. Dvorak D, Fenton AA. Toward a proper estimation of phase-amplitude coupling in neural oscillations. J Neurosci Methods (2014) 225:42-56. doi:10.1016/ j.jneumeth.2014.01.002

31. Lachaux JP, Rodriguez E, Martinerie J, Varela FJ. Measuring phase synchrony in brain signals. Hum Brain Mapp (1999) 8:194-208. doi:10.1002/(SICI)10970193(1999) 8:4<194::AID-HBM4>3.0.CO;2-C

32. Wiest MC, Nicolelis MA. Behavioral detection of tactile stimuli during 7-12 Hz cortical oscillations in awake rats. Nat Neurosci (2003) 6:913-4. doi:10.1038/ nn1107

33. Xanthopoulos P, Rebennack S, Liu CC, Zhang J, Holmes GL, Uthman BM, et al. A novel wavelet based algorithm for spike and wave detection in absence epilepsy. Bioinformatics and Bioengineering (BIBE) 2010 IEEE International Conference (2010). 
34. Semba K, Szechtman H, Komisaruk BR. Synchrony among rhythmical facial tremor, neocortical 'alpha' waves, and thalamic non-sensory neuronal bursts in intact awake rats. Brain Res (1980) 195:281-98. doi:10.1016/0006-8993(80) 90065-7

35. Gomora JC, Daud AN, Weiergraber M, Perez-Reyes E. Block of cloned human T-type calcium channels by succinimide antiepileptic drugs. Mol Pharmacol (2001) 60:1121-32. doi:10.1124/mol.60.5.1121

36. Mitchell KJ, O’Donnell P, Durstewitz D, Fenton AA, Gingrich JA, Gordon JA, et al. A Framework for the Use of Models in Schizophrenia. In: Silverstein SM, Moghaddam B, Wykes T, editors. Schizophrenia: Evolution and Synthesis. Cambridge, MA: MIT Press (2013). p. 212-26.

37. Patil ST, Zhang L, Martenyi F, Lowe SL, Jackson KA, Andreev BV, et al. Activation of mGlu2/3 receptors as a new approach to treat schizophrenia: a randomized Phase 2 clinical trial. Nat Med (2007) 13:1102-7. doi:10.1038/nm1007-1264

38. Duncan GE, Moy SS, Lieberman JA, Koller BH. Typical and atypical antipsychotic drug effects on locomotor hyperactivity and deficits in sensorimotor gating in a genetic model of NMDA receptor hypofunction. Pharmacol Biochem Behav (2006) 85:481-91. doi:10.1016/j.pbb.2006.09.017

39. Sun T, Hu G, Li M. Repeated antipsychotic treatment progressively potentiates inhibition on phencyclidine-induced hyperlocomotion, but attenuates inhibition on amphetamine-induced hyperlocomotion: relevance to animal models of antipsychotic drugs. Eur J Pharmacol (2009) 602:334-42. doi:10.1016/j.ejphar. 2008.11.036

40. Zhong J, Chuang SC, Bianchi R, Zhao W, Lee H, Fenton AA, et al. BC1 regulation of metabotropic glutamate receptor-mediated neuronal excitability. J Neurosci (2009) 29:9977-86. doi:10.1523/JNEUROSCI.3893-08.2009

41. Zhong J, Chuang SC, Bianchi R, Zhao W, Paul G, Thakkar P, et al. Regulatory $\mathrm{BC} 1 \mathrm{RNA}$ and the fragile $\mathrm{X}$ mental retardation protein: convergent functionality in brain. PLoS One (2010) 5:e15509. doi:10.1371/journal.pone.0015509

42. Carpenter WT Jr. How the diagnosis of schizophrenia impeded the advance of knowledge (and what to do about it). In: Silverstein SM, Moghaddam B, Wykes T, editors. Schizophrenia: Evolution and Synthesis. Cambridge, MA: MIT Press (2013). p. 49-62.

43. MacDonald AW. What kind of a thing is schizophrenia? Specific causation and general failure models. In: Silverstein SM, Moghaddam B, Wykes T, editors. Schizophrenia: Evolution and Synthesis. Cambridge, MA: MIT Press (2013). p. 25-48.

44. Hebb DO. The Organization of Behavior: A Neuropsychological Theory. New York: Wiley (1949).

45. Phillips WA, Singer W. In search of common foundations for cortical computation. Behav Brain Sci (1997) 20:657-83. doi:10.1017/S0140525X9700160X

46. Kelemen E, Fenton AA. Dynamic grouping of hippocampal neural activity during cognitive control of two spatial frames. PLoS Biol (2010) 8:e1000403. doi:10.1371/journal.pbio.1000403

47. Kelemen E, Fenton AA. Key features of human episodic recollection in the crossepisode retrieval of rat hippocampus representations of space. PLoS Biol (2013) 11:e1001607. doi:10.1371/journal.pbio.1001607

48. Steriade M, McCormick DA, Sejnowski TJ. Thalamocortical oscillations in the sleeping and aroused brain. Science (1993) 262:679-85. doi:10.1126/science. 8235588

49. Panayiotopoulos C. Absence epilepsies. In: Engel J Jr, Pedley TA, editors. Epilepsy: A Comprehensive Textbook. Philadelphia: Lippincott-Raven Publishers (1997). p. 2327-46.

50. Buzsaki G, Bickford RG, Ponomareff G, Thal LJ, Mandel R, Gage FH. Nucleus basalis and thalamic control of neocortical activity in the freely moving rat. $J$ Neurosci (1988) 8:4007-26.

51. Hosford DA, Clark S, Cao Z, Wilson WA Jr, Lin FH, Morrisett RA, et al. The role of GABAB receptor activation in absence seizures of lethargic (lh/lh) mice. Science (1992) 257:398-401. doi:10.1126/science.1321503

52. Neymotin SA, Lee H, Fenton AA, Lytton WW. Interictal EEG discoordination in a rat seizure model. J Clin Neurophysiol (2010) 27:438-44. doi:10.1097/WNP. 0b013e3181fe059e

53. Endo K, Hori T, Abe S, Asada T. Alterations in GABA(A) receptor expression in neonatal ventral hippocampal lesioned rats: comparison of prepubertal and postpubertal periods. Synapse (2007) 61:357-66. doi:10.1002/syn.20393

54. Francois J, Ferrandon A, Koning E, Angst MJ, Sandner G, Nehlig A. Selective reorganization of GABAergic transmission in neonatal ventral hippocampal-lesioned rats. Int J Neuropsychopharmacol (2009) 12:1097-110. doi:10.1017/S1461145709009985

55. Lipska BK, Lerman DN, Khaing ZZ, Weickert CS, Weinberger DR. Gene expression in dopamine and GABA systems in an animal model of schizophrenia: effects of antipsychotic drugs. Eur J Neurosci (2003) 18:391-402. doi:10.1046/j.1460-9568.2003.02738.x

56. O'Donnell P, Lewis BL, Weinberger DR, Lipska BK. Neonatal hippocampal damage alters electrophysiological properties of prefrontal cortical neurons in adult rats. Cereb Cortex (2002) 12:975-82. doi:10.1093/cercor/12.9.975

57. Lewis BL, O’Donnell P. Ventral tegmental area afferents to the prefrontal cortex maintain membrane potential 'up' states in pyramidal neurons via $\mathrm{D}(1)$ dopamine receptors. Cereb Cortex (2000) 10:1168-75. doi:10.1093/cercor/10. 12.1168

58. Kohler C, Schwarcz R. Comparison of ibotenate and kainate neurotoxicity in rat brain: a histological study. Neuroscience (1983) 8:819-35. doi:10.1016/03064522(83)90013-1

59. Babb TL, Pereira-Leite J, Mathern GW, Pretorius JK. Kainic acid induced hippocampal seizures in rats: comparisons of acute and chronic seizures using intrahippocampal versus systemic injections. Ital J Neurol Sci (1995) 16:39-44. doi:10.1007/BF02229073

60. Zimmerman FT, Burgemeister BB. A new drug for petit mal epilepsy. Neurology (1958) 8:769-75. doi:10.1212/WNL.8.10.769

61. Heathfield KW, Jewesbury EC. Treatment of petit mal with ethosuximide. $\mathrm{Br}$ Med J (1961) 2:565-7. doi:10.1136/bmj.2.5251.565

62. Goren MZ, Onat F. Ethosuximide: from bench to bedside. CNS Drug Rev (2007) 13:224-39. doi:10.1111/j.1527-3458.2007.00009.x

63. Coulter DA, Huguenard JR, Prince DA. Specific petit mal anticonvulsants reduce calcium currents in thalamic neurons. Neurosci Lett (1989) 98:74-8. doi:10.1016/0304-3940(89)90376-5

64. Coulter DA, Huguenard JR, Prince DA. Differential effects of petit mal anticonvulsants and convulsants on thalamic neurones: GABA current blockade. Br J Pharmacol (1990) 100:807-13. doi:10.1111/j.1476-5381.1990. tb14095.x

65. Schnitzler A, Gross J. Normal and pathological oscillatory communication in the brain. Nat Rev Neurosci (2005) 6:285-96. doi:10.1038/nrn1650

66. Lewis DA, Hashimoto T, Volk DW. Cortical inhibitory neurons and schizophrenia. Nat Rev Neurosci (2005) 6:312-24. doi:10.1038/nrn1648

67. Moghaddam B. Bringing order to the glutamate chaos in schizophrenia. Neuron (2003) 40:881-4. doi:10.1016/S0896-6273(03)00757-8

68. Beasley CL, Reynolds GP. Parvalbumin-immunoreactive neurons are reduced in the prefrontal cortex of schizophrenics. Schizophr Res (1997) 24:349-55. doi:10.1016/S0920-9964(96)00122-3

69. Woo TU, Miller JL, Lewis DA. Schizophrenia and the parvalbumincontaining class of cortical local circuit neurons. Am J Psychiatry (1997) 154: 1013-5.

70. Guidotti A, Auta J, Davis JM, Di-Giorgi-Gerevini V, Dwivedi Y, Grayson DR, et al. Decrease in reelin and glutamic acid decarboxylase67 (GAD67) expression in schizophrenia and bipolar disorder: a postmortem brain study. Arch Gen Psychiatry (2000) 57:1061-9. doi:10.1001/archpsyc.57.11.1061

71. Volk DW, Austin MC, Pierri JN, Sampson AR, Lewis DA. Decreased glutamic acid decarboxylase67 messenger RNA expression in a subset of prefrontal cortical gamma-aminobutyric acid neurons in subjects with schizophrenia. Arch Gen Psychiatry (2000) 57:237-45. doi:10.1001/archpsyc.57.3.237

72. Olney JW, Newcomer JW, Farber NB. NMDA receptor hypofunction model of schizophrenia. J Psychiatr Res (1999) 33:523-33. doi:10.1016/S0022-3956(99) 00029-1

73. Javitt DC. Glutamatergic theories of schizophrenia. Isr J Psychiatry Relat Sci (2010) 47:4-16

74. Lisman JE, Pi HJ, Zhang Y, Otmakhova NA. A thalamo-hippocampal-ventral tegmental area loop may produce the positive feedback that underlies the psychotic break in schizophrenia. Biol Psychiatry (2010) 68:17-24. doi:10.1016/j. biopsych.2010.04.007

75. Llinas RR, Ribary U, Jeanmonod D, Kronberg E, Mitra PP. Thalamocortical dysrhythmia: a neurological and neuropsychiatric syndrome characterized by magnetoencephalography. Proc Natl Acad Sci U S A (1999) 96:15222-7. doi:10.1073/pnas.96.26.15222

76. Uslaner JM, Smith SM, Huszar SL, Pachmerhiwala R, Hinchliffe RM, Vardigan JD, et al. T-type calcium channel antagonism produces antipsychotic-like effects 
and reduces stimulant-induced glutamate release in the nucleus accumbens of rats. Neuropharmacology (2010) 62(3):1413-21. doi:10.1016/j.neuropharm. 2010.11.015

77. Talley EM, Cribbs LL, Lee JH, Daud A, Perez-Reyes E, Bayliss DA. Differential distribution of three members of a gene family encoding low voltage-activated (T-type) calcium channels. J Neurosci (1999) 19:1895-911.

78. O’Donnell P, Grace AA. Synaptic interactions among excitatory afferents to nucleus accumbens neurons: hippocampal gating of prefrontal cortical input. $J$ Neurosci (1995) 15:3622-39.

79. Grace AA. Gating of information flow within the limbic system and the pathophysiology of schizophrenia. Brain Res Brain Res Rev (2000) 31:330-41. doi:10.1016/S0165-0173(99)00049-1

80. Enyeart JJ, Biagi BA, Mlinar B. Preferential block of T-type calcium channels by neuroleptics in neural crest-derived rat and human C cell lines. Mol Pharmacol (1992) 42:364-72.

81. Santi CM, Cayabyab FS, Sutton KG, McRory JE, Mezeyova J, Hamming KS, et al. Differential inhibition of T-type calcium channels by neuroleptics. J Neurosci (2002) 22:396-403.

82. Choi KH, Rhim H. Inhibition of recombinant Ca(v)3.1 (alpha(1G)) T-type calcium channels by the antipsychotic drug clozapine. Eur J Pharmacol (2010) 626:123-30. doi:10.1016/j.ejphar.2009.09.035

83. Smoller JW, Craddock N, Kendler K, Lee PH, Neale BM, Nurnberger JI, et al. Identification of risk loci with shared effects on five major psychiatric disorders: a genome-wide analysis. Lancet (2013) 381:1371-9. doi:10.1016/S01406736(12)62129-1

84. Lieberman JA, Stroup TS, McEvoy JP, Swartz MS, Rosenheck RA, Perkins DO, et al. Effectiveness of antipsychotic drugs in patients with chronic schizophrenia. N Engl J Med (2005) 353:1209-23. doi:10.1056/NEJMoa051688

85. Keefe RS, Bilder RM, Davis SM, Harvey PD, Palmer BW, Gold JM, et al. Neurocognitive effects of antipsychotic medications in patients with chronic schizophrenia in the CATIE trial. Arch Gen Psychiatry (2007) 64:633-47. doi:10.1001/archpsyc.64.6.633

86. Bymaster FP, Calligaro DO, Falcone JF, Marsh RD, Moore NA, Tye NC, et al. Radioreceptor binding profile of the atypical antipsychotic olanzapine. Neuropsychopharmacology (1996) 14:87-96. doi:10.1016/0893-133X(94) 00129-N

87. Horacek J, Bubenikova-Valesova V, Kopecek M, Palenicek T, Dockery C, Mohr P, et al. Mechanism of action of atypical antipsychotic drugs and the neurobiology of schizophrenia. CNS Drugs (2006) 20:389-409. doi:10.2165/ 00023210-200620050-00004

88. Amann BL, Pogarell O, Mergl R, Juckel G, Grunze H, Mulert C, et al. EEG abnormalities associated with antipsychotics: a comparison of quetiapine, olanzapine, haloperidol and healthy subjects. Hum Psychopharmacol (2003) 18:641-6. doi:10.1002/hup. 537

89. Pillmann F, Schlote K, Broich K, Marneros A. Electroencephalogram alterations during treatment with olanzapine. Psychopharmacology (Berl) (2000) 150:216-9. doi:10.1007/s002130000426

90. Wichniak A, Szafranski T, Wierzbicka A, Waliniowska E, Jernajczyk W. Electroencephalogram slowing, sleepiness and treatment response in patients with schizophrenia during olanzapine treatment. J Psychopharmacol (2006) 20:80-5. doi:10.1177/0269881105056657

91. Centorrino F, Price BH, Tuttle M, Bahk WM, Hennen J, Albert MJ, et al. EEG abnormalities during treatment with typical and atypical antipsychotics. Am J Psychiatry (2002) 159:109-15. doi:10.1176/appi.ajp.159.1.109

92. Hubl D, Kleinlogel H, Frolich L, Weinandi T, Maurer K, Holstein W, et al. Multilead quantitative electroencephalogram profile and cognitive evoked potentials (P300) in healthy subjects after a single dose of olanzapine. Psychopharmacology (Berl) (2001) 158:281-8. doi:10.1007/s002130100861

Conflict of Interest Statement: The authors declare that the research was conducted in the absence of any commercial or financial relationships that could be construed as a potential conflict of interest.

Received: 01 September 2013; accepted: 29 January 2014; published online: 14 February 2014.

Citation: Lee H, Dvorak D and Fenton AA (2014) Targeting neural synchrony deficits is sufficient to improve cognition in a schizophrenia-related neurodevelopmental model. Front. Psychiatry 5:15. doi: 10.3389/fpsyt.2014.00015

This article was submitted to Schizophrenia, a section of the journal Frontiers in Psychiatry.

Copyright (c) 2014 Lee, Dvorak and Fenton. This is an open-access article distributed under the terms of the Creative Commons Attribution License (CC BY). The use, distribution or reproduction in other forums is permitted, provided the original author(s) or licensor are credited and that the original publication in this journal is cited, in accordance with accepted academic practice. No use, distribution or reproduction is permitted which does not comply with these terms. 